\title{
The expression patterns and prognostic significance of pleckstrin homology-like domain family A (PHLDA) in lung cancer and malignant mesothelioma
}

\author{
Camila M. Baldavira ${ }^{1}$, Juliana Machado-Rugolo ${ }^{1,2}$, Tabatha G. Prieto ${ }^{1}$, Daniel R. Bastos ${ }^{3,4}$, \\ Marcelo Balancin ${ }^{1}$, Alexandre M. Ab'Saber ${ }^{1}$, Lygia B. Yaegashi ${ }^{1}$, Paola C. Souza ${ }^{1}$, Cecilia Farhat ${ }^{1}$, \\ Teresa Y. Takagaki ${ }^{5}$, Maria Ap. Nagai ${ }^{3,4}$, Vera L. Capelozzi ${ }^{1}$ \\ ${ }^{1}$ Department of Pathology, University of São Paulo Medical School (USP), São Paulo, Brazil; ${ }^{2}$ Health Technology Assessment Center (NATS), \\ Clinical Hospital (HCFMB), Medical School of São Paulo State University (UNESP), Botucatu, Brazil; ${ }^{3}$ Department of Radiology and Oncology, \\ Medical School of São Paulo State University (UNESP), Brazil; ${ }^{4}$ Laboratory of Molecular Genetics, Center for Translational Research in Oncology, \\ Cancer Institute of São Paulo (ICESP), São Paulo, Brazil; ${ }^{5}$ Division of Pneumology, Instituto do Coração (Incor), University of São Paulo Medical \\ School (USP), São Paulo, Brazil \\ Contributions: (I) Conception and design: MA Nagai, VL Capelozzi; (II) Administrative support: VL Capelozzi; (III) Provision of study materials or \\ patients: M Balancin, AM Ab'Saber, LB Yaegashi, PC Souza, TY Takagaki; (IV) Collection and assembly of data: CM Baldavira, J Machado-Rugolo, \\ TG Prieto, DR Bastos; (V) Data analysis and interpretation: CM Baldavira, J Machado-Rugolo, TG Prieto, DR Bastos; (VI) Manuscript writing: All \\ authors; (VII) Final approval of manuscript: All authors. \\ Correspondence to: Prof. Dr. Vera L. Capelozzi. Department of Pathology, University of São Paulo Medical School (USP), Av Dr Arnaldo 455, Room \\ 1143, São Paulo, São Paulo State 01246-903, Brazil. Email: vera.capelozzi@fm.usp.br.
}

Background: Pleckstrin homology domain family A (PHLDA) genes play important roles in cancer cellular processes, including inhibiting Akt activation, repressing growth factor signaling, inhibiting the negative feedback of EGFR/ErbB2 signaling cells, and inducing apoptosis. However, the prognostic significance of PHLDA in non-small cell lung cancer (NSCLC) and malignant pleural mesothelioma (MM) remains unclear. The present study investigates the associations between PHLDA expression patterns and their prognostic value in lung adenocarcinoma (LUAD) and MM.

Methods: We analyzed PHLDA family members at the genomic level in silico to explore their mRNA expression pattern and predictive significance in LUAD and MM. We then created a PHLDA-drug interaction network and a protein-protein interaction (PPI) network using different databases. Finally, we immunohistochemically assessed the protein expression of each PHLDA family member on tissue microarrays (TMAs) in both LUAD and MM cohorts with long-term follow-up.

Results: While PHLDA1 mRNA expression in both LUAD and MM was lower than that of normal tissue, PHLDA2 mRNA was significantly overexpressed in LUAD, and PHLDA3 mRNA was overexpressed in MM. In NSCLC, both low PHLDA1 mRNA expression and high PHLDA3 mRNA expression correlated with worse overall survival (OS) $(\mathrm{P}<0.01)$, whereas high $P H L D A 2$ mRNA expression was associated with better OS $(\mathrm{P}<0.01)$. In MM, patients presenting high PHLDA1 and PHLDA2 mRNA expression had poor OS $(\mathrm{P}=0.01$ and $\mathrm{P}<0.01$, respectively). In addition, the PHLDA-drug interaction network indicated that several common drugs could potentially modulate PHLDA expression, and the PPI network suggested that PHLDA1 interacts with Notch family members, whereas PHLDA3 interacts with TP53. Our results also showed that the expression of PHLDA2 and PHLDA3 was significantly higher in LUAD and MM than that of PHLDA1 $(\mathrm{P}<0.05)$ and was associated with the risk of death. While patients with PHLDA2 $>85.09$ cells $/ \mathrm{mm}^{2}$ had a low risk of death $(\mathrm{P}=0.01)$ and a median survival time of 48 months, those with PHLDA $3<70.38$ cells $/ \mathrm{mm}^{2}$ had a high risk of death $(\mathrm{P}=0.03)$ and a median survival time of 34 months.

$\wedge$ ORCID: 0000-0002-5364-7305. 
Conclusions: We shed light on the role of the PHLDA family as promising predictive biomarkers and potential therapeutic targets in LUAD and MM.

Keywords: Morphometry; data mining; malignant mesothelioma; lung adenocarcinoma; pleckstrin homology-like domain family A (PHLDA)

Submitted Sep 16, 2020. Accepted for publication Dec 03, 2020.

doi: 10.21037/jtd-20-2909

View this article at: http://dx.doi.org/10.21037/jtd-20-2909

\section{Introduction}

Non-small cell lung cancer (NSCLC) and malignant pleural mesothelioma (MM) are pathologies that share the common fact that both are potentially highly aggressive tumors, and their prognosis is highly dependent on the histological subtype, lymph node status, and the pathological TNM stage of the disease (1-4). In addition, these characteristics end up influencing the decision-making decision regarding the therapeutic strategy. For both, surgery is the standard treatment for patients with early disease, however, the overall 5 -year survival rate remains below 50\% (1-3).

Today, the major difference between these pathologies is the broader range of therapeutic possibilities available for NSCLC, while MM does not yet have a broad arsenal. Therefore, new biomarkers are much necessary that could assist in more individualized treatment for both pathologies. Since conventional therapeutic protocols have been limited in their effects on treatment outcomes, innovative strategies for treating NSCLC and MM are now being explored, especially those that are molecularly targeted.

The pleckstrin homology-like domain, family A (PHLDA) is a family of genes consisting of 3 members: PHLDA1, PHLDA2, and PHLDA3 (5). The genes in the PHLDA family have a similar organization, involving one coding exon and one noncoding exon separated by a small intron (6). All three genes encode for $\mathrm{PH}$ domaincontaining proteins. These proteins share high similarity within their $\mathrm{PH}$ domains and are detectable using immunohistochemistry screening with variable sensitivity and specificity (5). Cumulative evidence has shown that PHLDA is a family of tumor oncogenes that play important roles in cancer cellular processes, including competing with Akt signaling (7-9), repressing growth factor signaling (10), inhibiting negative feedback of EGFR/ErbB2 signaling cells (8), inducing apoptosis (6), and recent reports suggest the PHLDA3 gene acts as a tumor suppressor in human neuroendocrine tumors $(11,12)$.
Thus, the current study seeks to support the clinical value of PHLDA genes as potential agnostic biomarkers and to point at new targetable therapeutic pathways for patients with NSCLC and MM. We comprehensively analyzed the expression patterns of PHLDA family genes and their prognostic value in LUAD and MM using in silico data-mining investigation methods combined with clinical immunohistochemistry on tissue microarrays.

We present the following article in accordance with the REMARK reporting checklist (available at http://dx.doi. org/10.21037/jtd-20-2909).

\section{Methods}

\section{PHLDA mRNA expression}

We used UALCAN (RRID:SCR_015827), a user-friendly web resource for analyzing data from The Cancer Genome Atlas (TCGA; RRID:SCR_003193) (13), to investigate the relative mRNA expression of PHLDA family genes in lung adenocarcinoma (LUAD) and normal samples. The mRNA expression level of PHLDA genes was normalized to transcript per million reads, and only a $\mathrm{P}$ value of no more than 0.01 , according to a Student's $t$-test, was to be significant. We selected the Genomic Spatial Event (GSE) database (14) and GSE51024 (15) downloaded from NCBI GEO to analyze the mRNA expression of PHLDA family genes in $M M$ and normal tissues according to adjusted $\mathrm{P}<0.01$ and having $\mid \log \mathrm{FCl}>2$ as the threshold. The Oncomine database (RRID:SCR_007834), the world's largest cancer microarray database and web-based data mining platform for extracting cancer gene information $(16,17)$, was then used to determine the differences in the mRNA expression of each PHLDA family member between different cancer specimens and normal controls. The $\mathrm{P}$ value for these differences in expression was established through a Student's $t$-test. We set the threshold at 2.0-fold change, $\mathrm{P}=0.05$, and top $10 \%$ gene rank. 
The prognostic significance value of the mRNA expression of PHLDA genes in NSCLC was evaluated using the Kaplan-Meier plotter database (RRID:SCR_018753) (18). Only validated probes were used to access the overall survival (OS), first progression (FPS), and post-progression survival (PPS) curves. Patient samples were divided into two cohorts, according to the median expression of each gene (high vs. low expression). The effects of different patterns of PHLDA expression on OS were estimated through univariate and multivariate Cox proportional hazards models, with or without adjustments for confounding factors. Variables including gender, TNM stage, and age were further adjusted during the evaluation. The Kaplan-Meier plotter database calculated the logrank $\mathrm{P}$ value and hazard ratio (HR) with $95 \%$ confidence intervals (CI). $\mathrm{P}<0.05$ was considered significant. The UCSC Xena (RRID:SCR_018938) (19) browser (http:// xena.ucsc.edu/) was used to analyze OS in Mesothelioma (TCGA, Firehose Legacy) data.

\section{Interaction networks}

We searched within the Comparative Toxicogenomics Database (CTD; RRID: SCR_006530) (20) for PHLDA genes in cases employing drugs or chemicals that could decrease/increase their mRNA or protein expression and used this data to create a PHLDA-drug interaction network. This network was then generated using Cytoscape (RRID:SCR_003032) (21). Finally, we used the Search Tool for the Retrieval of Interacting Genes/Proteins (STRING; RRID:SCR_005223) database (22) to explore the proteinprotein interaction (PPI) between PHLDA family members.

\section{PHLDA protein expression}

\section{Tissue samples}

Specimens were obtained from archived formalin-fixed paraffin-embedded (FFPE) histological sections of 102 primary tumors from patients with stage I-IV who had been treated at Clinicas Hospital of University of São Paulo Medical School (HC-FMUSP), the Heart Institute (InCor) and São Paulo Cancer Institute (ICESP) between 1995 and 2018. Their histologic diagnoses were reviewed and confirmed by two pathologists who specialize in lung cancer. The study included 41 LUAD and $61 \mathrm{MM}$ (epithelioid and sarcomatoid histotypes), placed in tissue microarrays (TMAs), none of which had received neoadjuvant therapy. Each TMA was constructed from the primary resected tumors using three $1.5 \mathrm{~mm}$ tissue cores obtained from the center, middle, and periphery of the most representative tumor areas that were selected and marked on hematoxylineosin (HE) stained slides. Normal liver and kidney tissues were used for control and slide orientation purposes. The previous histological assessment of each analysis area was performed to ensure that the tumor tissue (at least $85 \%$ malignant cells) was included in the selected intratumoral region. Twelve slides containing 1,197 tumor cores were evaluated.

The Internal Ethics Committees of all participant institutions approved this study protocol.

\section{Immunohistochemistry analysis}

Firstly, the immunostains were tested on 10 whole tissue sections and 18 TMA to ensure that the stain was evenly and not patchy. Four-micron-thick sequential whole tissue sections and TMA sections were stained with immunoperoxidase. The staining was performed with antibodies against PHLDA1 (sc6142, clone M-20, dilution 1:100; Santa Cruz Biotechnology, Santa Cruz, CA, EUA; RRID:AB_2163292), PHLDA2 (polyclonal antibody, CAT\#: TA344404, dilution 1:50; Origene, OriGene Technologies, Rockville, USA), PHLDA3 (polyclonal antibody, CAT\#: TA315261, dilution 1:100; Origene, OriGene Technologies, Rockville, USA) and tumor suppressor P53 (clone 3186-11, dilution 1:200; DAKO, Via Real Carpinteria, CA, USA). Cell expression of all markers was detected using a Novocastra Bond Polymer Refine Detection kit (Leica Microsystems) with a diaminobenzidine reaction to detect antibodies labeling and hematoxylin counterstaining. For PHLDAs expression, cytoplasmic with perinuclear accentuation and dots patterns were considered positive. Nuclear expression was considered positive for P53.

Immunohistochemical staining for the PHLDAs family showed homogeneous cytoplasmic staining with perinuclear accentuation and cytoplasmic staining in dots in all $10 \mathrm{MM}$ and 18 LUAD whole mount slides and tumor cores. In addition, immunohistochemical staining for PHLDA family antibodies showed homogeneous cytoplasmic staining with perinuclear accentuation and cytoplasmic staining in dots in 40 (40\%) MM and 60 (60\%) LUAD tumor cores (Figure S1).

\section{Image analysis quantification}

To measure the IHC expression of each different marker and quantify protein expression, the TMA slides were digitally scanned at $\times 40$ magnification using a Panoramic 
Table 1 Frequency of clinicopathologic characteristics of the patients included in the study

\begin{tabular}{lcc}
\hline Characteristic & $\begin{array}{c}\text { Lung } \\
\text { adenocarcinoma } \\
(\mathrm{N}=41)\end{array}$ & $\begin{array}{c}\text { Malignant } \\
\text { mesothelioma } \\
(\mathrm{N}=61)\end{array}$ \\
\hline $\begin{array}{l}\text { Age (years), median } \\
\text { Sex, n (\%) }\end{array}$ & 65 & 59 \\
Female & $17(41.5)$ & $18(29.5)$ \\
Male & $22(53.7)$ & $43(70.5)$ \\
Tumor stage ${ }^{\dagger}, \mathrm{n}(\%)$ & $17(41.5)$ & - \\
I & $14(34.1)$ & - \\
II & $5(12.2)$ & $61(100.0)$ \\
III & $5(12.2)$ & - \\
IV & & - \\
Nodal status†, n (\%) & & \\
N0 & $21(51.2)$ & - \\
N1 & $15(36.6)$ & \\
Status, n (\%) & $26(63.4)$ & - \\
Death & & \\
\hline
\end{tabular}

${ }^{\dagger}$, according to the International Association for the Study of Lung Cancer $(1,23,24) .{ }^{a}$, in 2 patients with LUAD we did not find information about your gender; ${ }^{\mathrm{b}}$, in 5 patients with LUAD we did not find information about the lymph nodes status.

250 whole slide scanner (3DHistech, Budapest, Hungary). The stained TMA sections were disarrayed within QuPath version 0.2.0-m429, 30 (Centre for Cancer Research \& Cell Biology, University of Edinburgh, Edinburgh, Scotland; RRID:SCR_018257), an open-source image analysis platform. All cores were evaluated during the scoring process to manually exclude invalid cores (less than $10 \%$ of tumor per core or artefacts).

The TMAs were quantified on QuPath using a simple automated, semi-assisted method. First, each scanned TMA slide underwent a series of automated evaluations, starting with a staining vector analysis, followed by total tissue area detection, tumor separation from non-tumor areas, and cellular detection. Next, we established the threshold of positivity for each one of our four markers through trial and error, and positive cells were submitted to validation by an expert pathologist before being applied to the full array. QuPath then exported the result of this analysis as the number of positive cells per $\mathrm{mm}^{2}$ of tissue.

Based on each marker's threshold of positivity, we generated a script used to automatically detect positive and negative cells in each slide and export the results in image format be validated by a pathologist who was blind to patient outcome. A membrane and cytoplasmic algorithm quantified PHLDA immunohistochemical expression, whereas a nuclear algorithm quantified P53 expression. The average number of positive nuclei was expressed as positive cell density per $\mathrm{mm}^{2}$ and used as the median value to which specific samples were compared. We refer to low expression as positive cell density equal or below the median expression level, and high expression as positive cell density above this median.

\section{Clinical data and outcome}

Clinical data of MM and LUAD patients was derived from an existing prospectively maintained database. Patient and tumor characteristics were retrospectively collected including age, sex, smoking history, tumor size, tumor stage $(1,23,24)$, and follow-up information for OS rates (Table 1). OS served as the primary outcome and was defined as the interval from surgery to death or last contact. Secondary outcomes included associations with clinicopathological factors.

\section{Statistical analysis}

According to protocols of the tools employed in the in silico analyses, the mRNA levels of PHLDA in NSCLC, MM, and normal tissues were analyzed in each individual dataset using the Student's $t$-test and the patients' OS distribution was estimated using the Kaplan-Meier method. A logrank test was performed to determine the difference in survival between the groups. For the clinical data, either the Chi-square test or the Fisher exact test was used to examine differences in categorical clinical variables, and the Wilcoxon rank-sum test and Kruskal-Wallis test were used to detect differences in continuous immunohistochemistry variables between groups of patients. A regression analysis of the risk of death (OR) was performed using univariate and multivariate Cox proportional hazards models. The criteria to include the variables in multivariate analysis was: (I) using the $\mathrm{P}$ value obtained in Cox's univariate analysis, or (II) using classically accepted criteria with impact on the risk of death and OS, namely staging and histological types, as co-dependent variables of the significant variables obtained in the univariate analysis. If no statistical differences were found for any clinicopathologic and immunohistochemistry variables, multivariate analysis will be performed to 

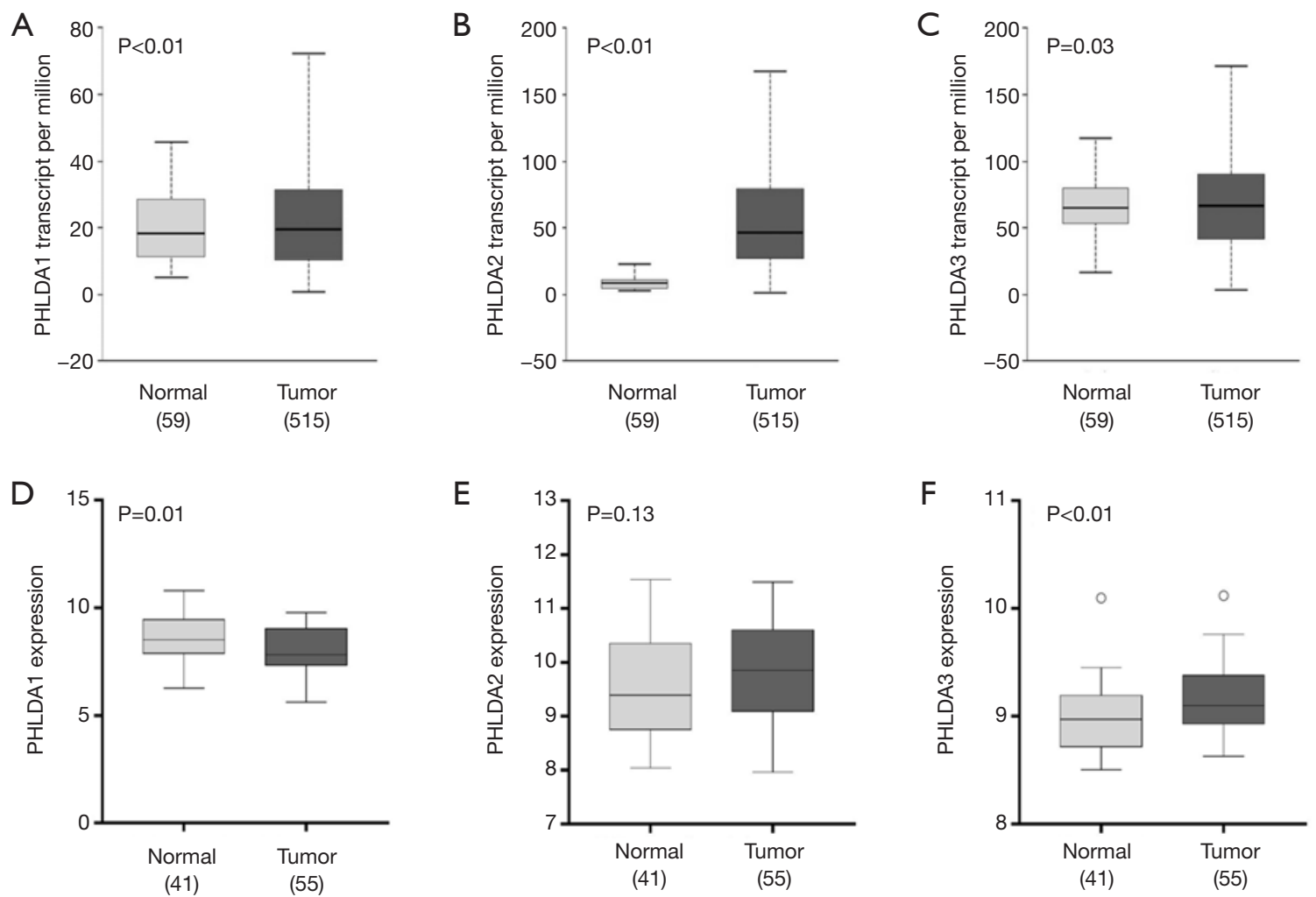

Figure 1 The relative expression of pleckstrin homology domain family A (PHLDA) genes in normal and lung adenocarcinoma (LUAD) tissues according to The Cancer Genome Atlas (TCGA) datasets and the relative expression of PHLDA genes in normal and malignant mesothelioma (MM) tissues according to GSE51024 raw data. (A) PHLDA1, (B) PHLDA2, and (C) PHLDA3 in LUAD subtype tissue, (D) PHLDA1, (E) PHLDA2, and (F) PHLDA3 in MM tissue. (A) All PHLDA genes were highly expressed in LUAD when compared to normal tissue. (D) $P H L D A 1$ gene expression in MM was lower than in normal tissue; (F) PHLDA3 gene expression was significantly higher than in normal tissue; (E) no statistical difference was found for PHLDA2 expression between MM and normal samples.

determine if the immunohistochemistry variables would depend on the control of the model (co-dependency) by the classically accepted variables with an impact on the risk of death and OS, such as staging and histological types.

The statistical software programs IBM SPSS (version 22; Armonk, NY, USA; RRID:SCR_002865) and S-Plus (version 8.04; TIBCO, Palo Alto, CA, USA) were used to perform the computations for all analyses. $\mathrm{P}$ value $<0.05$ was considered significant.

\section{Ethical statement}

The study was conducted in accordance with the Declaration of Helsinki (as revised in 2013). The study was approved by the national ethics committee of $\mathrm{n}^{\circ} 3.729 .622$ and individual consent for this retrospective analysis was waived.

\section{Results}

\section{In silico data mining}

\section{PHLDA mRNA expression}

The UALCAN analysis showed that PHLDA1, PHLDA2, and PHLDA3 mRNA expression levels were statistically significant in LUAD samples compared to normal tissues $(\mathrm{P}<0.01, \mathrm{P}<0.01$, and $\mathrm{P}=0.03$, respectively; Figure $1 A, B, C)$. There was a significant correlation between LUAD squamoid alveolar lineage type and PHLDA1 $(\mathrm{P}=0.02)$, PHLDA2 $(\mathrm{P}<0.01)$, and PHLDA3 $(\mathrm{P}<0.01)$ genes. No statistical differences were found for the other parameters (Table 2).

The GSE51024 raw data on MM showed that PHLDA1 mRNA expression was lower than that of normal tissue $(\mathrm{P}=0.01), P H L D A 2$ expression was not statistically significant $(\mathrm{P}=0.13)$, and $P H L D A 3$ expression was 
Table 2 Clinicopathological characteristics of the patients with lung cancer from TCGA database (Chi-square test; $\mathrm{P}$ value $<0.05$ )

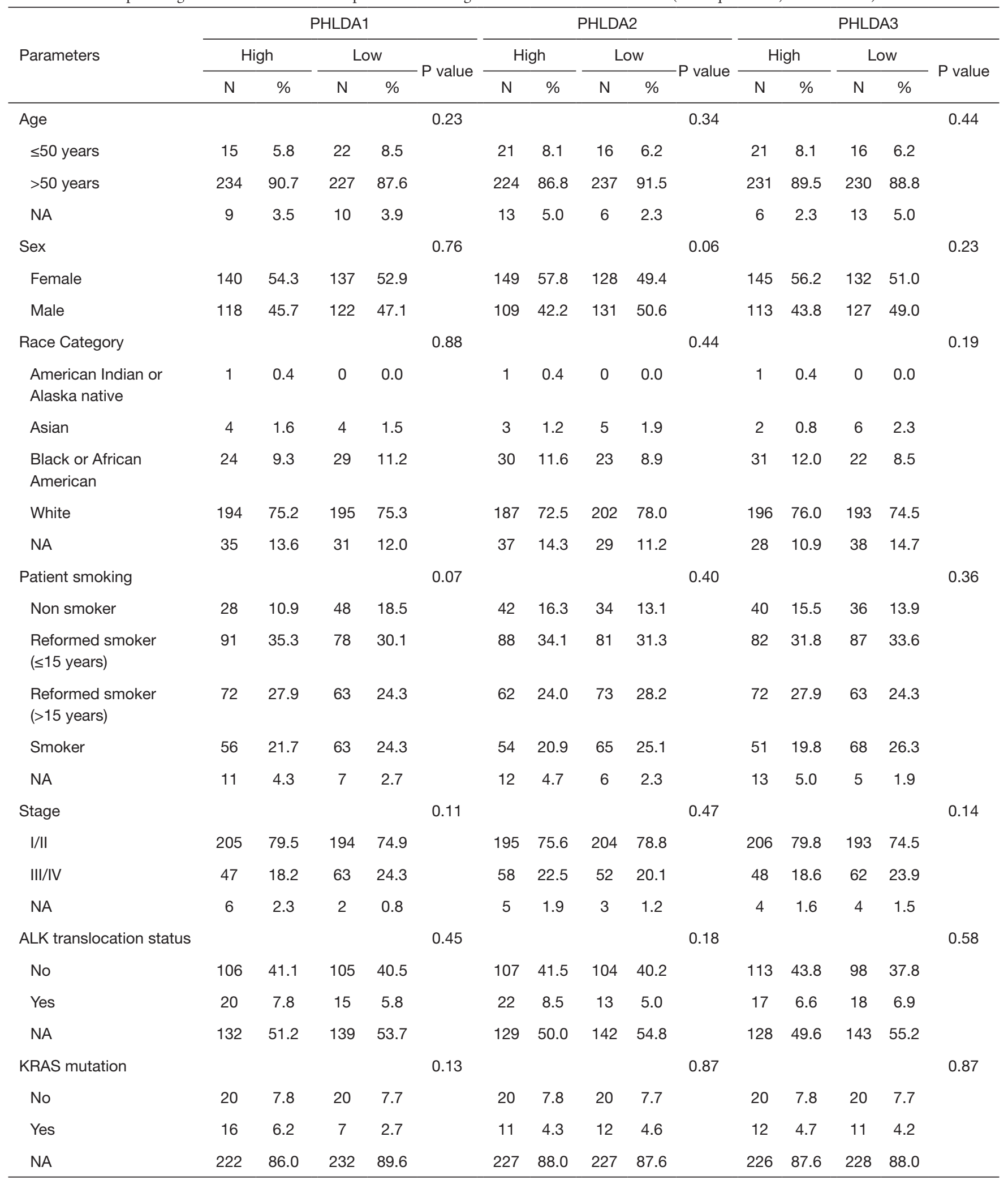

Table 2 (continued) 
Table 2 (continued)

\begin{tabular}{|c|c|c|c|c|c|c|c|c|c|c|c|c|c|c|c|}
\hline \multirow{2}{*}{ Parameters } & \multicolumn{5}{|c|}{ PHLDA1 } & \multicolumn{5}{|c|}{ PHLDA2 } & \multicolumn{5}{|c|}{ PHLDA3 } \\
\hline & $\mathrm{N}$ & $\%$ & $\mathrm{~N}$ & $\%$ & $P$ value & $\mathrm{N}$ & $\%$ & $\mathrm{~N}$ & $\%$ & $P$ value & $\mathrm{N}$ & $\%$ & $\mathrm{~N}$ & $\%$ & $P$ value \\
\hline Expression subtype $^{\dagger}$ & & & & & 0.02 & & & & & $<0.01$ & & & & & $<0.01$ \\
\hline Bronchioid & 48 & 18.6 & 41 & 15.8 & & 36 & 14.0 & 53 & 20.5 & & 56 & 21.7 & 33 & 12.7 & \\
\hline Squamoid & 49 & 19.0 & 29 & 11.2 & & 60 & 23.3 & 18 & 6.9 & & 43 & 16.7 & 35 & 13.5 & \\
\hline NA & 136 & 52.7 & 151 & 58.3 & & 142 & 55.0 & 145 & 56.0 & & 140 & 54.3 & 147 & 56.8 & \\
\hline
\end{tabular}

${ }^{\dagger}$, according to Song et al. [2018] (25). PHLDA, pleckstrin homology domain family A; NA, not available.

significantly increased compared to normal samples $(\mathrm{P}<0.01)$ (Figure $1 D, E, F)$. Lower PHLDA3 mRNA expression was associated with lymph node status $(\mathrm{P}=0.02)$, tumor size $(\mathrm{P}=0.01)$, and TP53 mutational status $(\mathrm{P}<0.01)$. No correlation was found between PHLDA1 and PHLDA2 expression and clinicopathological characteristics (Table 3).

To further validate and cross-examine the observations made in the TCGA and GEO databases, the Oncomine data was used to evaluate the differential mRNA levels of the PHLDA genes in both LUAD and MM. The mRNA expression levels of PHLDA1, PHLDA2, and PHLDA3 were collected from 457, 436, and 360 different types of tumor studies, respectively (Figure S2). Of these, 90 studies showed a statistical difference for the PHLDA1 gene, 56 for the PHLDA2 gene, and 6 for the PHLDA3 gene. From these data groups, we selected three datasets: Hou Lung (203476_at), Selamat Lung (ILMN_1687978), and Su Lung (217996_at) to investigate the expression of these genes in LUAD histotype and normal tissues (Figure S3).

The Hou Lung and Selamat datasets showed no significant difference in the expression of PHLDA1 mRNA (fold change $=1.18$ and -1.03 ) (Figure S3A,B). However, the Su dataset indicated a trend for PHLDA1 mRNA underexpression in LUAD (fold change $=-1.71$ ) (Figure $\mathrm{S} 3 \mathrm{C}$ ). In all three databases, PHLDA2 mRNA was overexpressed (fold change $=2.21,2.39$, and 3.63, respectively) in LUAD compared to normal tissue (Figure S3D,E,F). Conversely, none of the datasets showed changes in the mRNA levels of PHLDA3 (fold change $=-1.14,1.29$, and 1.03, respectively) (Figure S3G,H,I). Finally, the Gordon Mesothelioma dataset was used to assess PHLDA mRNA expression in MM (Figure S4), and neither PHLDA1 nor PHLDA3 mRNA showed a significantly different expression in tumor tissue compared to normal tissue (fold change $=-1.09$ and 1.25 , respectively) (Figure S4A,C). PHLDA2, however, trended toward underexpression in MM (fold change $=-1.92$ ) (Figure S4B).

\section{Prognostic significance of $P H L D A$ mRNA expression}

A Kaplan-Meier plotter analysis was used to find the correlation between PHLDA mRNA levels and OS, FPS, and PPS in NSCLC patients (Figure 2). Low PHLDA1 mRNA expression was correlated with worse OS (HR $=0.77$; 95\% CI: 0.63-0.94; $\mathrm{P}<0.01$; Figure $2 A)$, FPS ( $\mathrm{HR}=0.66$; 95\% CI: 0.47-0.93; $\mathrm{P}=0.02$; Figure $2 D$ ), and PPS (HR $=0.43 ; 95 \%$ CI: $0.26-0.72 ; \mathrm{P}<0.01$, Figure $2 G)$; whereas high $P H L D A 2$ mRNA expression was significantly associated with better OS (HR $=0.84$; 95\% CI: 0.74-0.96; $\mathrm{P}<0.01$; Figure $2 B)$, though not with FPS (HR $=1.14 ; 95 \%$ CI: $0.92-1.40 ; \mathrm{P}=0.24$; Figure $2 E$ ) or PPS (HR $=0.82$; $\mathrm{CI}$ : $0.62-1.08 ; \mathrm{P}=0.16$; Figure $2 H$ ). Finally, patients with high PHLDA3 mRNA expression had poor OS (HR $=1.25 ; 95 \%$ CI: $1.1-1.42 ; \mathrm{P}<0.01$; Figure $2 C$ ), FPS (HR $=1.78 ; 95 \% \mathrm{CI}$ : $1.41-2.24 ; \mathrm{P}<0.01$; Figure $2 F$ ), and PPS (HR $=1.37 ; 95 \%$ CI: $1.02-1.83 ; \mathrm{P}=0.03$; Figure 21 ).

Next, we correlated OS with clinicopathological data (Figure S5). Low PHLDA1 mRNA expression was significantly related to worse survival in male patients ( $\mathrm{HR}=0.56 ; 95 \%$ CI: $0.37-0.86 ; \mathrm{P}<0.01)$, female patients $(\mathrm{HR}=0.52 ; 95 \% \mathrm{CI}: 0.34-0.77 ; \mathrm{P}<0.01)$ and smokers (HR $=0.37 ; 95 \%$ CI: $0.2-0.67 ; \mathrm{P}<0.01$ ) (Figure S5A,B,D); high PHLDA2 mRNA expression was significantly related to poor survival in male patients ( $\mathrm{HR}=1.52$; $95 \% \mathrm{CI}$ : $1.08-2.14$; $\mathrm{P}=0.01)$ and in non-smokers ( $\mathrm{HR}=4.42 ; 95 \% \mathrm{CI}: 1.83-$ 10.67, $\mathrm{P}<0.01$ ) (Figure S5E,G); and high PHLDA3 mRNA expression was correlated with short survival in male patients ( $\mathrm{HR}=1.46 ; 95 \%$ CI: 1.01-2.11; $\mathrm{P}=0.04$ ), female patients (HR 
Table 3 Clinicopathological characteristics of the patients with malignant mesothelioma from TCGA database (Chi-square test; P value $<0.05$ )

\begin{tabular}{|c|c|c|c|c|c|c|c|c|c|c|c|c|c|c|c|}
\hline \multirow{2}{*}{ Parameters } & \multicolumn{5}{|c|}{ PHLDA1 } & \multicolumn{4}{|c|}{ PHLDA2 } & \multicolumn{6}{|c|}{ PHLDA3 } \\
\hline & $\mathrm{N}$ & $\%$ & $\mathrm{~N}$ & $\%$ & $P$ value & $\mathrm{N}$ & $\%$ & $\mathrm{~N}$ & $\%$ & $P$ value & $\mathrm{N}$ & $\%$ & $\mathrm{~N}$ & $\%$ & $P$ value \\
\hline Age & & & & & 0.23 & & & & & 0.05 & & & & & 0.05 \\
\hline$\leq 50$ years & 2 & 4.7 & 5 & 11.4 & & 6 & 14.0 & 1 & 2.3 & & 6 & 14.0 & 1 & 2.3 & \\
\hline Race category & & & & & 1.00 & & & & & 1.00 & & & & & 1.00 \\
\hline Asian & 1 & 2.3 & 0 & 0.0 & & 0 & 0.0 & 1 & 2.3 & & 1 & 2.3 & 0 & 0.0 & \\
\hline $\begin{array}{l}\text { Black or African } \\
\text { American }\end{array}$ & 0 & 0.0 & 1 & 2.3 & & 0 & 0.0 & 1 & 2.3 & & 0 & 0.0 & 1 & 2.3 & \\
\hline Male & 37 & 86.0 & 34 & 77.3 & & 36 & 83.7 & 35 & 79.5 & & 32 & 74.4 & 39 & 88.6 & \\
\hline Tumor size & & & & & 0.72 & & & & & 0.60 & & & & & 0.01 \\
\hline 1 & 7 & 16.3 & 7 & 15.9 & & 6 & 14.0 & 8 & 18.2 & & 3 & 7.0 & 11 & 25.0 & \\
\hline 2 & 12 & 27.9 & 14 & 31.8 & & 16 & 37.2 & 10 & 22.7 & & 18 & 41.9 & 8 & 18.2 & \\
\hline 3 & 18 & 41.9 & 14 & 31.8 & & 15 & 34.9 & 17 & 38.6 & & 18 & 41.9 & 14 & 31.8 & \\
\hline 4 & 5 & 11.6 & 8 & 18.2 & & 6 & 14.0 & 7 & 15.9 & & 4 & 9.3 & 9 & 20.5 & \\
\hline$T X$ & 1 & 2.3 & 1 & 2.3 & & 0 & 0.0 & 2 & 4.5 & & 0 & 0.0 & 2 & 4.5 & \\
\hline Negative & 27 & 62.8 & 30 & 68.2 & & 27 & 62.8 & 30 & 68.2 & & 33 & 76.7 & 24 & 54.5 & \\
\hline Positive & 1 & 2.3 & 2 & 4.5 & & 2 & 4.7 & 1 & 2.3 & & 2 & 4.7 & 1 & 2.3 & \\
\hline$M X$ & 15 & 34.9 & 12 & 27.3 & & 14 & 32.6 & 13 & 29.5 & & 8 & 18.6 & 19 & 43.2 & \\
\hline Stage & & & & & 0.61 & & & & & 0.53 & & & & & 0.05 \\
\hline 1 & 6 & 14.0 & 4 & 9.1 & & 6 & 14.0 & 4 & 9.1 & & 3 & 7.0 & 7 & 15.9 & \\
\hline II & 6 & 14.0 & 10 & 22.7 & & 10 & 23.3 & 6 & 13.6 & & 12 & 27.9 & 4 & 9.1 & \\
\hline III & 24 & 55.8 & 21 & 47.7 & & 20 & 46.5 & 25 & 56.8 & & 23 & 53.5 & 22 & 50.0 & \\
\hline IV & 7 & 16.3 & 9 & 20.5 & & 7 & 16.3 & 9 & 20.5 & & 5 & 11.6 & 11 & 25.0 & \\
\hline TP53 status & & & & & 0.39 & & & & & 0.73 & & & & & $<0.01$ \\
\hline Wild type & 38 & 88.4 & 36 & 81.8 & & 36 & 83.7 & 38 & 86.4 & & 41 & 95.3 & 33 & 75.0 & \\
\hline Mutated & 5 & 11.6 & 8 & 18.2 & & 7 & 16.3 & 6 & 13.6 & & 2 & 4.7 & 11 & 25.0 & \\
\hline
\end{tabular}

PHLDA, pleckstrin homology domain family A. 
A
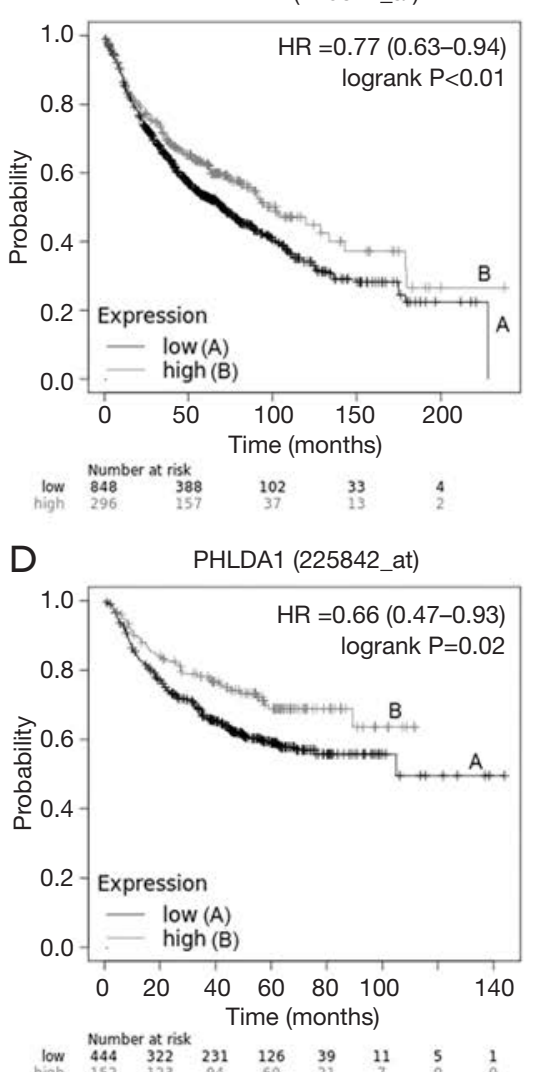

G

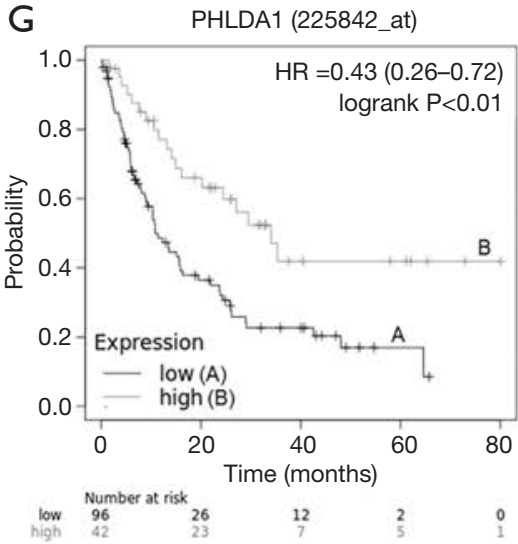

B
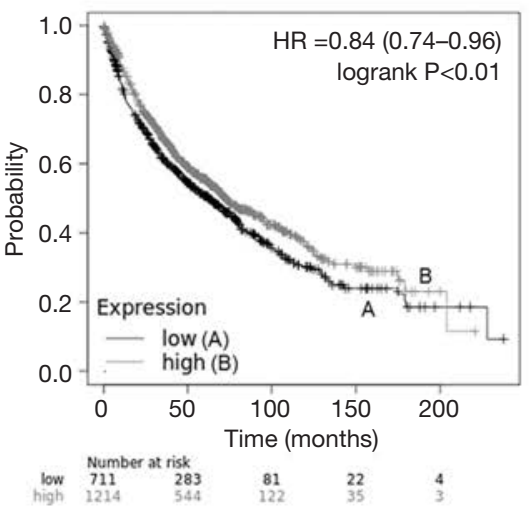

E

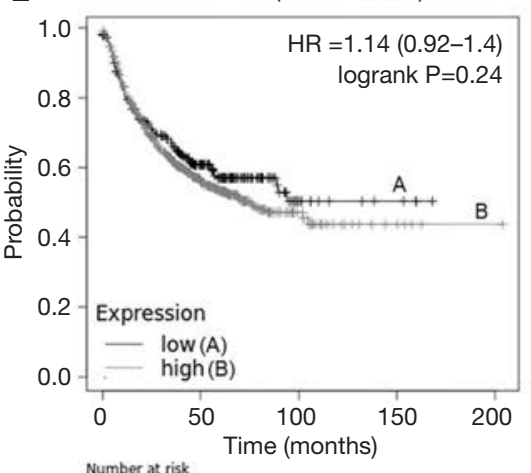

low ${ }_{\text {high }}$ N

$\mathrm{H}$

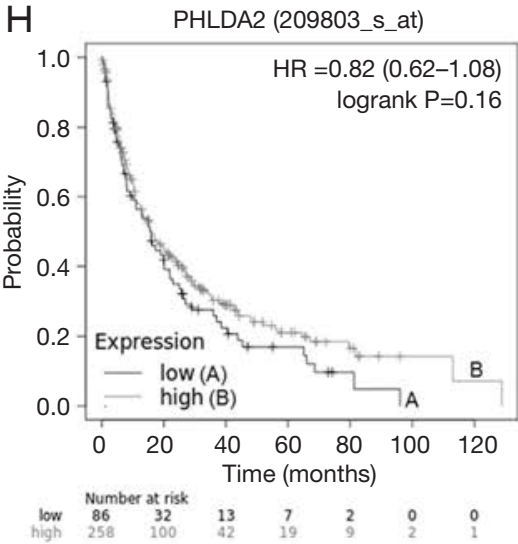

C

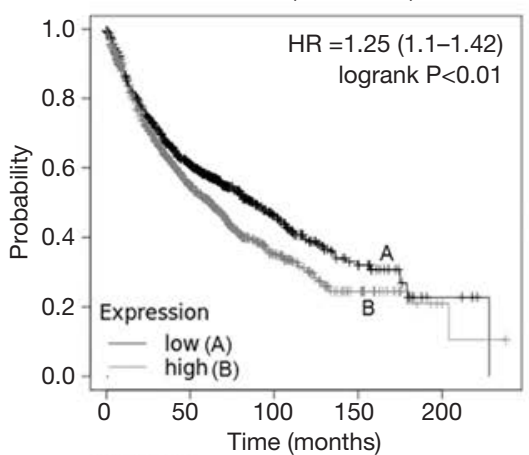

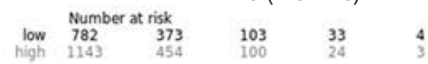

$\mathrm{F}$
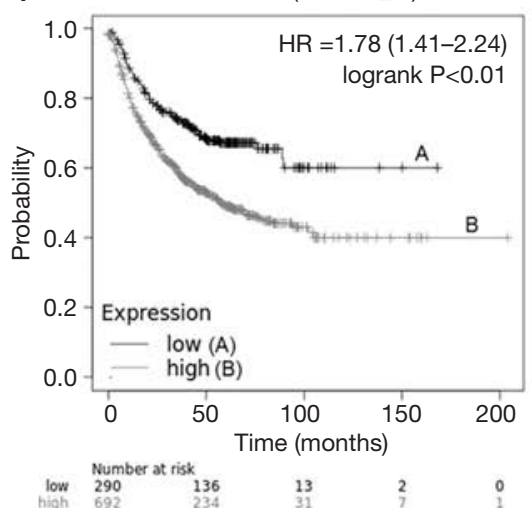

I

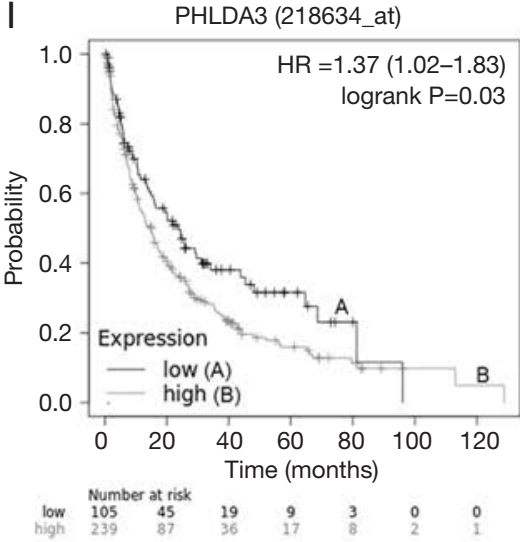

Figure 2 This Kaplan Meier survival curve shows the correlation between pleckstrin homology domain family A (PHLDA) genes expression and overall survival (OS), first progression survival (FPS) and post progression survival (PPS) in lung cancer patients. (A) Low expression of the PHLDA1 gene was correlated with worse OS (HR =0.77, 95\% CI: 0.63-0.94; $\mathrm{P}<0.01$ ); (B) high expression of the PHLDA2 gene was significantly associated with better OS (HR $=0.84,95 \%$ CI: 0.74-0.96; $\mathrm{P}<0.01)$; (C) high expression of the PHLDA3 gene also correlated with worse OS (HR $=1.25,95 \%$ CI: 1.1-1.42; $\mathrm{P}<0.01)$; (D) low expression of the PHLDA1 gene showed a significant correlation with worse FPS (HR $=0.66,95 \%$ CI: 0.47-0.93; P=0.02); (E) PHLDA2 gene expression was not statistically significant on FPS (HR $=1.14,95 \%$ CI: 0.92-1.40; $\mathrm{P}=0.24$ ); (F) high expression of the PHLDA3 gene was significantly correlated with worse FPS (HR $=1.78,95 \%$ CI: 1.41-2.24; $\mathrm{P}<0.01$ ); (G) low expression of the PHLDA1 gene showed a significant correlation with worse PPS (HR $=0.43,95 \%$ CI: $0.26-0.72 ; \mathrm{P}<0.01$ ); (H) PHLDA2 gene expression was not statistically significant on PPS (HR $=0.82,95 \%$ CI: $0.62-1.08 ; \mathrm{P}=0.16$ ); (I) high expression of the PHLDA3 gene was significantly correlated with worse PPS (HR =1.37, 95\% CI: 1.02-1.83; P=0.03). 

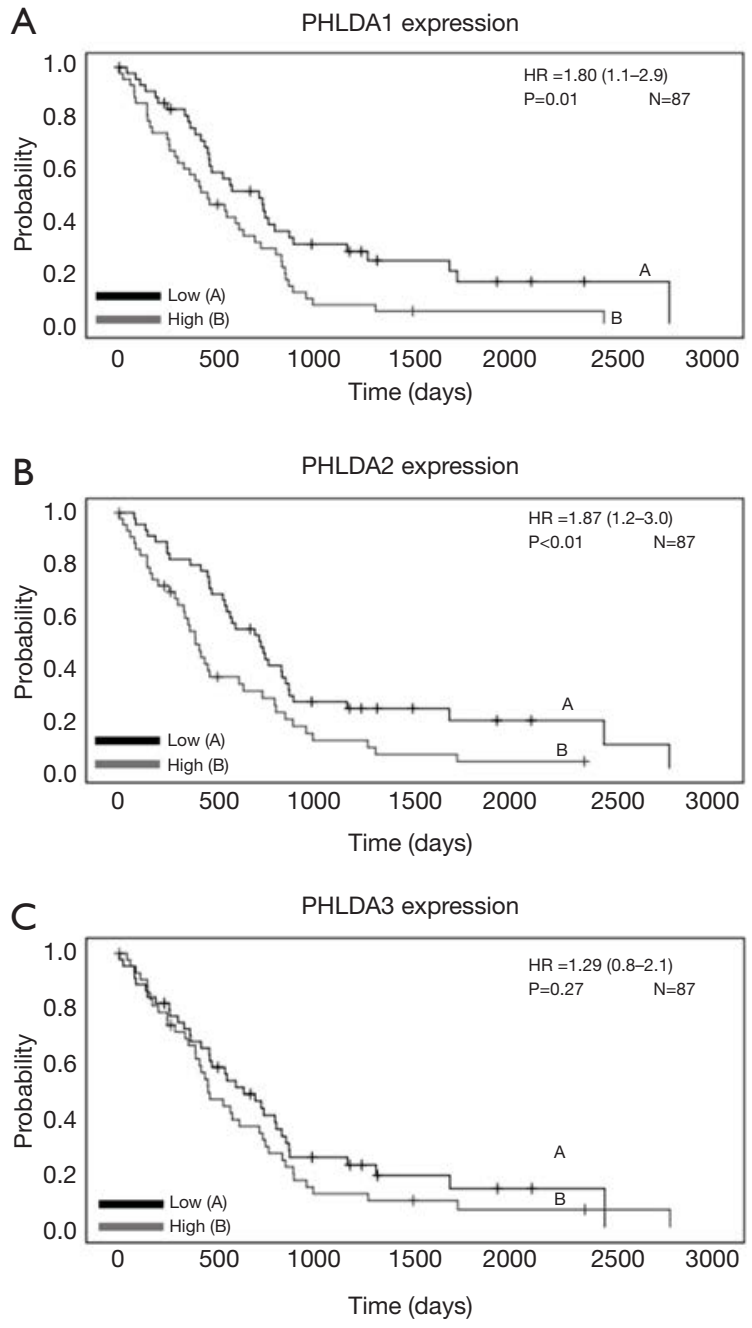

Figure 3 This Kaplan Meier survival curve shows the correlation between pleckstrin homology domain family A (PHLDA) genes expression and overall survival (OS) in malignant mesothelioma patients according to the The Cancer Genome Atlas (TCGA) Firehose Legacy datasets. (A) High expression of the PHLDA1 (HR=1.80, 95\% CI: 1.1-2.9; $\mathrm{P}=0.01)$ and (B) PHLDA2 (HR=1.87, 95\% CI: $1.2-3.0 ; \mathrm{P}<0.01)$ genes was significant correlated with worse OS; (C) PHLDA3 gene expression was not correlated with survival (HR =1.29, 95\% CI: $0.8-2.1 ; \mathrm{P}=0.27)$.

=1.86; 95\% CI: 1.24-2.8; $\mathrm{P}<0.01)$, non-smokers $(\mathrm{HR}=2.82$; 95\% CI: $1.26-6.3, \mathrm{P}<0.01)$, and smokers $(\mathrm{HR}=1.83 ; 95 \%$ CI: 1.13-2.95; $\mathrm{P}=0.01$ ) (Figure S5I,J,K,L).

The Kaplan-Meier curves in MM ( $\mathrm{n}=87$ samples) were generated using Mesothelioma (TCGA, Firehose Legacy) data from the UCSC Xena tool (Table S1). While patients with MM presenting high PHLDA1 and PHLDA2 mRNA expression had poor OS (HR =1.80; 95\% CI: 1.1-2.9; $\mathrm{P}=0.01$, and $\mathrm{HR}=1.87 ; 95 \% \mathrm{CI}: 1.2-3.0 ; \mathrm{P}<0.01$, respectively; Figure $3 A, B)$, their PHLDA3 mRNA expression showed no correlation with survival ( $\mathrm{HR}=1.29$; 95\% CI: 0.8-2.1; $\mathrm{P}=0.27$; Figure 3C). A multivariate Cox analysis that controlled for age, sex, lymph node metastases, stage, and TP53 confirmed that PHLDA1 and PHLDA2 mRNA expression were independent predictors of worse OS (HR =1.64; 95\% CI: 1.0-2.6; $\mathrm{P}=0.04$ and $\mathrm{HR}=1.71,95 \% \mathrm{CI}: 1.1-2.7 ; \mathrm{P}=0.03$; Table S1).

\section{Interaction network}

We used the Comparative Toxicogenomics Database to investigate the possibility of interaction and modulation between PHLDA genes and potential chemical drugs. Our PHLDA-drug interaction network indicated that a number of commonly used drugs could modulate the mRNA or protein expression of PHLDA. Specifically, chemotherapy agents, including cisplatin, potentially decrease PHLDA1 levels; whereas carcinogenic substances, such as tetrachlorodibenzodioxin, were able to increase the expression of all PHLDA family members (Figure 4).

Figure 5 shows the significant PHLDA-PPI network that was designed using the STRING database. Its molecular organization can be visualized as a network of differentially connected nodes. Each node stands for a protein and its edges represent dynamic interactions. The PHLDA1-PPI network had 31 nodes and 100 edges $(\mathrm{P}=2.06 \mathrm{e}-08)$, and its most significant biological processes and pathways were associated with positive regulation of the transcription of both the Notch receptor target (GO:0007221; Purple color) and Notch signaling pathway (GO:0007219; Red color) (Figure 5A). The PHLDA2-PPI network had 11 nodes and 40 edges $(\mathrm{P}=1.42 \mathrm{e}-12)$ and was related to the activity of cyclindependent protein serine/threonine kinase (GO:0004693; Red color) and the p53 signaling pathway (hsa04115; Purple color) (Figure 5B). Finally, the PHLDA3-PPI network had 21 nodes and 82 edges $(\mathrm{P}=8.31 \mathrm{e}-12)$ and was mainly associated with signal transduction by p53 class mediator (Figure 5C).

Taken together, the above in silico results suggest that the PHLDA genes may be reliable prognostic indicators for malignant thoracic tumors, including MM and NSCLC.

\section{PHLDA protein expression}

\section{Protein expression and histotypes}

PHLDA protein expression was assessed using immunohistochemistry on tissue microarrays (TMAs) in a well-characterized cohort of 41 LUAD and 61 
A

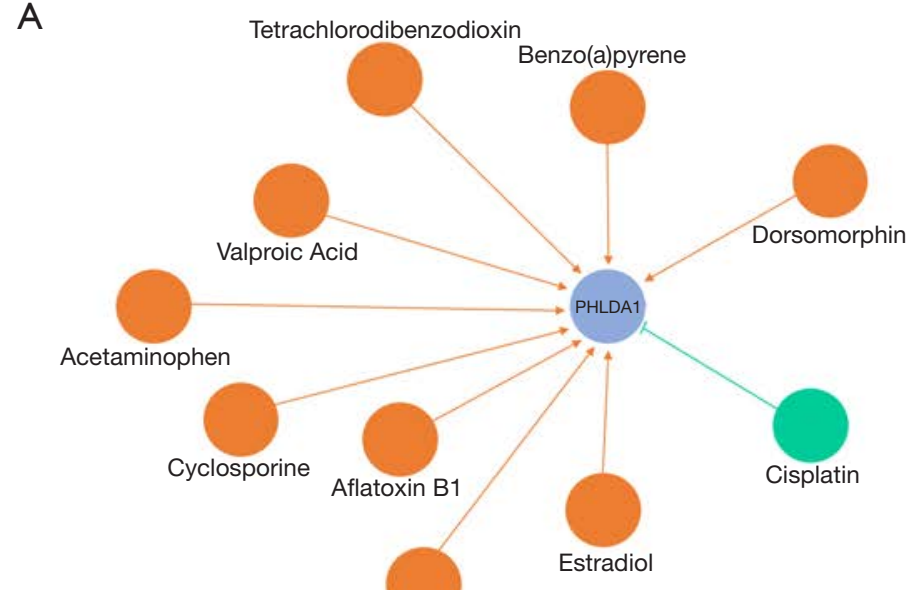

4-(5-benzo(1,3)dioxol-5-yl-4-pyridin-2-yl-1H-imidlazol-2-yl) benzamide

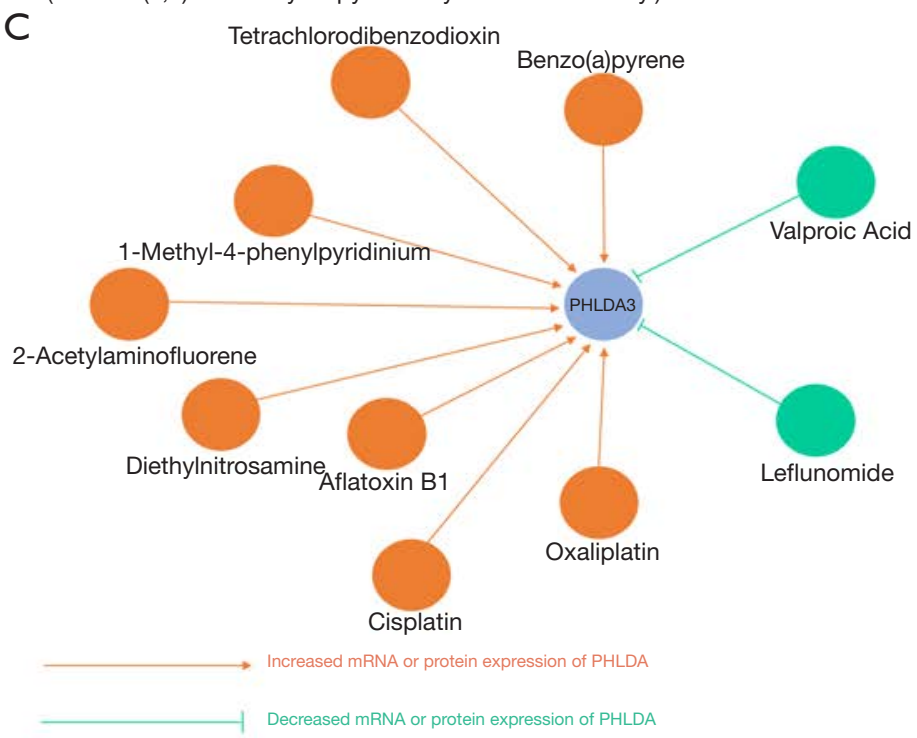

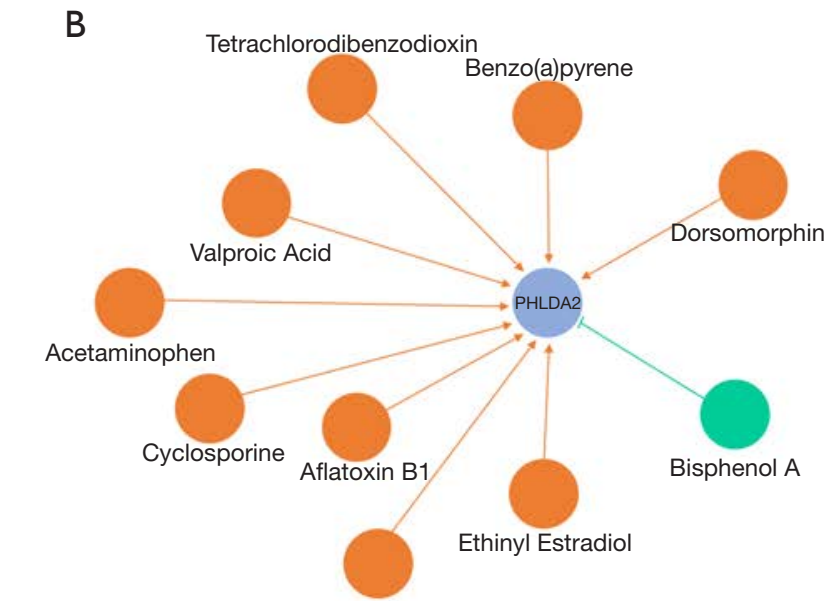

4-(5-benzo(1,3)dioxol-5-yl-4-pyridin-2-yl-1H-imidlazol-2-yl) benzamide

Figure 4 The PHLDA-drug interaction network obtained from the Comparative Toxicogenomics Database. (A) PHLDA1-drug interaction network; (B) PHLDA2-drug interaction network; (C) PHLDA3-drug interaction network. This network shows that several common drugs could potentially modulate the mRNA or protein expression of PHLDA. PHLDA, pleckstrin homology domain family A.

MM. Figure 6 brings representative images of LUAD and MM stained by HE (Figure $6 A, B$ ), and subjected to immunohistochemistry for PHLDA1 (Figure 6C,D), PHLDA2 (Figure 6E,F), PHLDA3 (Figure 6G,H) and P53 (Figure 6I,f). The PHLDA1 staining created a cytoplasmic expression in dots pattern in mild number of $\mathrm{MM}$ and LUAD malignant cells (Figure $6 C, D$ ). In contrast, the PHLDA2 staining showed strong and diffuse expression in cytoplasm with perinuclear accentuation in numerous MM cells (Figure $6 E$ ), while a mild number of LUAD cells showed a predominance of cytoplasmic dots pattern (Figure
6F). Regarding PHLDA3 staining, a mild and diffuse cytoplasmic expression in numerous MM cells (Figure 6G), comparing to the cytoplasmic with perinuclear accentation expression in mild number of LUAD (Figure 6H). TP53 staining was predominantly found in LUAD (Figure 67), and almost absent in MM (Figure 6I).

Table 4 shows the protein expression frequency of PHLDAs and P53 in 41 LUAD and 61 MM samples. The expression of PHLDA2 and PHLDA3 was significantly higher in LUAD (median 88.16 cells $/ \mathrm{mm}^{2}$ and 84.90 cells $/ \mathrm{mm}^{2}$, respectively) than in $\mathrm{MM}$ (median $79.51 \mathrm{cells} / \mathrm{mm}^{2}$ and 
A

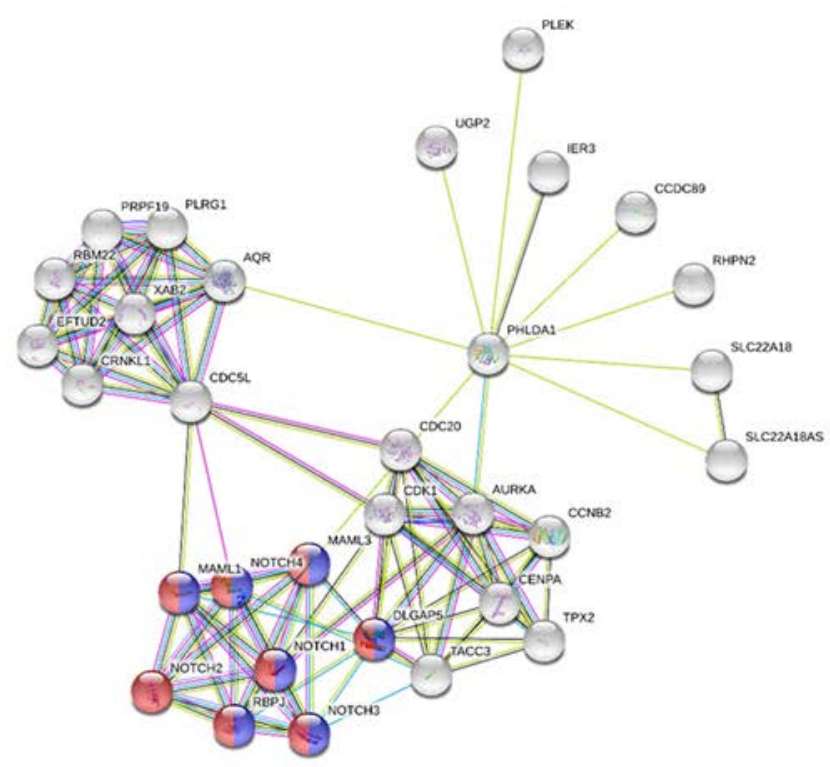

C

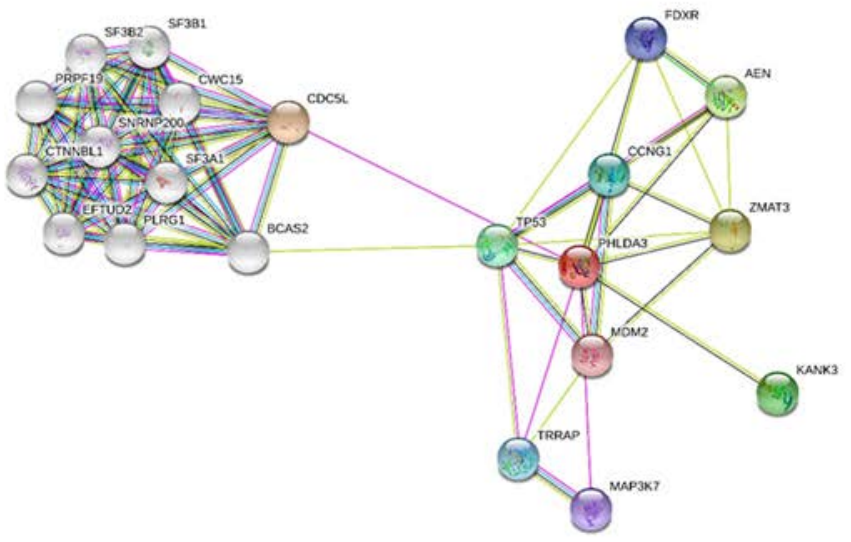

B

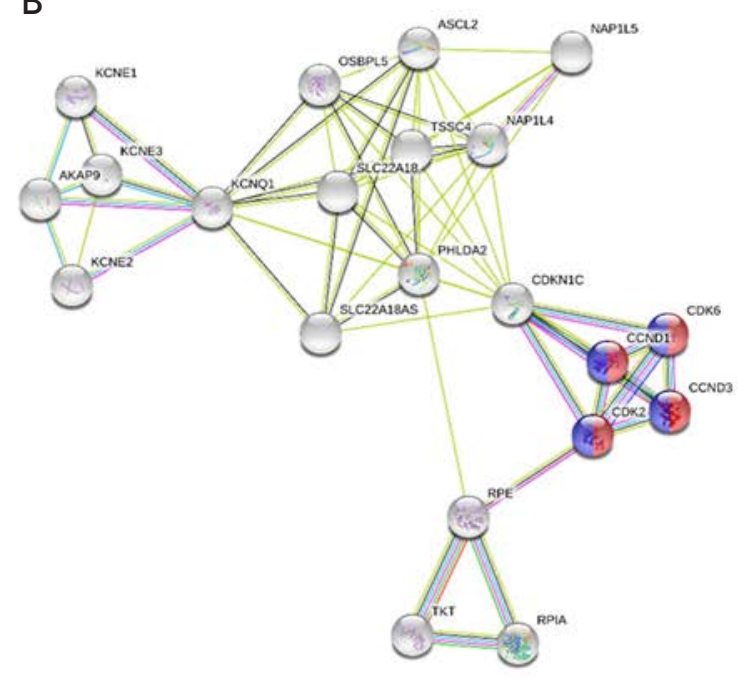

Figure 5 Cluster analysis of the PPI network. (A) PHLDA1-PPI network. (B) PHLDA2-PPI network, and (C) PHLDA3-PPI network. PHLDA, pleckstrin homology domain family A; PPI, protein-protein interaction.

67.80 cells $/ \mathrm{mm}^{2}$ respectively). PHLDA1 expression was not statistically significant $(\mathrm{P}=0.37)$. 23 cases of LUAD $(56.1 \%)$ and 31 cases of $M M(50.8 \%)$ presented values above the median expression of PHLDA1 $(\mathrm{P}=0.49)$. For PHLDA2 protein expression, 26 cases (63.4\%) of LUAD and 28 cases (45.9\%) of MM showed higher cell density than the median $(\mathrm{P}=0.02)$, and the $\mathrm{PHLDA} 3$ protein was expressed above the median in 33 cases $(80.5 \%)$ of LUAD, and 27 cases $(44.3 \%)$ of $M M(\mathrm{P}<0.01)$.

\section{Protein expression and clinicopathological parameters}

The demographic data and clinicopathological characteristics of the LUAD and MM cohorts were stratified according to PHLDA and P53 expression (Table 5). Specimens from 29 (28.4\%) patients with LUAD presented higher expression of PHLDA3 compared to 22 (21.6\%) patients with MM $(\mathrm{P}<0.01)$. Among patients in clinical stage IV, PHLDA3 expression was lower in 41 patients, and high in 25 patients $(\mathrm{P}<0.01)$. A significant correlation was demonstrated among $31(31.6 \%)$ patients with high PHLDA1 expression $(\mathrm{P}=0.03)$, high PHLDA2 expression $(\mathrm{P}=0.01)$, high PHLDA3 expression $(\mathrm{P}<0.01)$ and high $\mathrm{P} 53$ protein expression (median expression value of 2.9 cells $/ \mathrm{mm}^{2}$ ). No statistical differences were found for the other parameters. 


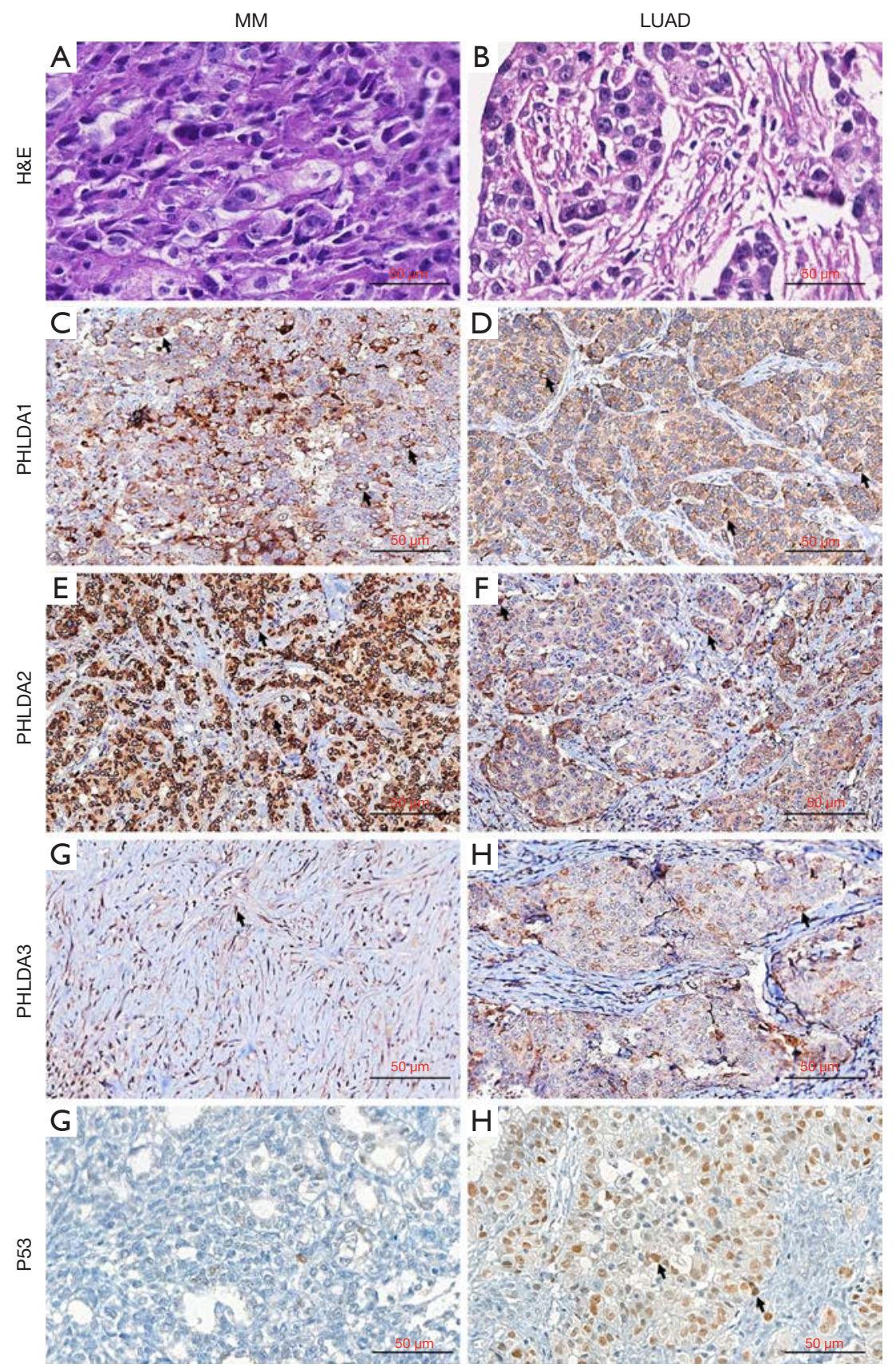

Figure 6 PHLDA protein expression using immunohistochemistry on tissue microarrays (TMAs) in MM and LUAD. H\&E staining of an epithelioid MM section/showing blocks of epithelioid cells infiltrating sparse desmoplasic stroma (A); H\&E staining of a representative solid subtype LUAD composed of cohesive and poor differentiated malignant cells, aggregated in blocks and immersed in exuberant desmoplasic stroma (B). Immunohistochemistry: PHLDA1 staining of mild number of epithelioid MM cells (arrows, C) and LUAD cells (arrows, D); PHLDA2 with strong and diffuse cytoplasmic staining with perinuclear accentuation in numerous MM malignant cells (arrows, E), contrasting with diffuse cytoplasmic staining in a mild number of LUAD malignant cells (arrows, F); PHLDA3 with diffuse cytoplasmic expression in MM malignant cells (arrows, G), and cytoplasmic staining with perinuclear accentuation in a mild number of LUAD malignant cells (arrows, H); strong and diffuse P53 nuclear staining in LUAD (arrows, J) and weak, almost absent, in MM (I). PHLDA, pleckstrin homology domain family A; H\&E, hematoxylin \& eosin; LUAD, lung adenocarcinoma; MM, malignant mesothelioma. 
Table 4 Median densities of PHLDAs and TP53 according to histological type (N=102) (Mann-Whitney test; P value <0.05)

\begin{tabular}{|c|c|c|c|c|c|}
\hline Protein (cells/mm²) & \multicolumn{2}{|c|}{ Malignant mesothelioma } & \multicolumn{2}{|c|}{ Lung adenocarcinoma } & $P$ value \\
\hline PHLDA1 & 52.76 & 51.47 & 55.49 & 56.74 & 0.37 \\
\hline PHLDA2 & 74.73 & 79.51 & 86.17 & 88.16 & 0.01 \\
\hline PHLDA3 & 64.99 & 67.80 & 80.71 & 84.90 & $<0.01$ \\
\hline
\end{tabular}

PHLDA, pleckstrin homology domain family A.

Table 5 PHLDA and p53 protein expression and clinicopathologic characteristics of the patients with lung cancer and malignant mesothelioma (Chi-square test; $\mathrm{P}$ value $<0.05$ )

\begin{tabular}{|c|c|c|c|c|c|c|c|c|c|}
\hline Characteristic & \multicolumn{3}{|c|}{ PHLDA1 (cell/mm²) } & \multicolumn{3}{|c|}{ PHLDA2 (cell/mm²) } & \multicolumn{3}{|c|}{ PHLDA3 (cell/mm²) } \\
\hline Age (years) & & & 0.42 & & & 0.11 & & & 0.11 \\
\hline$>61.5$ & 27 (27.6\%) & $22(22.4 \%)$ & & 29 (29.6\%) & $20(20.4 \%)$ & & $29(29.6 \%)$ & $20(20.4 \%)$ & \\
\hline$\leq 61.5$ & $22(22.4 \%)$ & 27 (27.6\%) & & $20(20.4 \%)$ & $29(29.6 \%)$ & & $20(20.4 \%)$ & 29 (29.6\%) & \\
\hline Female & $13(13.0 \%)$ & $22(22.0 \%)$ & & 17 (17.0\%) & $18(18.0 \%)$ & & $13(13.0 \%)$ & $22(22.0 \%)$ & \\
\hline Male & $37(37.0 \%)$ & $28(28.0 \%)$ & & $33(33.0 \%)$ & $32(32.0 \%)$ & & $37(37.0 \%)$ & $28(28.0 \%)$ & \\
\hline Tumor type & & & 0.42 & & & 0.22 & & & $<0.01$ \\
\hline LUAD & $18(17.6 \%)$ & $23(22.5 \%)$ & & 17 (16.7\%) & 24 (23.5\%) & & $12(11.8 \%)$ & 29 (28.4\%) & \\
\hline I & $7(7.0 \%)$ & $8(8.0 \%)$ & & 7 (7.0\%) & $8(8.0 \%)$ & & $3(3.0 \%)$ & $12(12.0 \%)$ & \\
\hline II & $7(7.0 \%)$ & $7(7.0 \%)$ & & $5(5.0 \%)$ & $9(9.0 \%)$ & & $5(5.0 \%)$ & $9(9.0 \%)$ & \\
\hline III & $1(1.0 \%)$ & $4(4.0 \%)$ & & $2(2.0 \%)$ & $3(3.0 \%)$ & & $1(1.0 \%)$ & $4(4.0 \%)$ & \\
\hline IV & 35 (35.0\%) & $31(31.0 \%)$ & & $36(36.0 \%)$ & $30(30.0 \%)$ & & $41(41.0 \%)$ & 25 (25.0\%) & \\
\hline Status $^{a}$ & & & 0.36 & & & 0.20 & & & 0.58 \\
\hline Alive & $13(13.3 \%)$ & 20 (20.4\%) & & $12(12.2 \%)$ & $21(21.4 \%)$ & & 17 (17.3\%) & $16(16.3 \%)$ & \\
\hline Dead & 34 (34.7\%) & $28(28.6 \%)$ & & $35(35.7 \%)$ & 27 (27.6\%) & & $30(30.6 \%)$ & 32 (32.7\%) & \\
\hline$>2.9 \mathrm{cell} / \mathrm{mm}^{2}$ & 18 (18.4\%) & 31 (31.6\%) & & $18(18.4 \%)$ & 31 (31.6\%) & & $18(18.4 \%)$ & 31 (31.6\%) & \\
\hline
\end{tabular}

${ }^{\dagger}$, according to the International Association for the Study of Lung Cancer $(1,23,24)$. ${ }^{a}, 3$ cases could not define the patient's status. PHLDA, pleckstrin homology domain family A; LUAD, lung adenocarcinoma; MM, malignant mesothelioma. 
Table 6 The univariate and multivariate analysis employed a Cox proportional hazards model

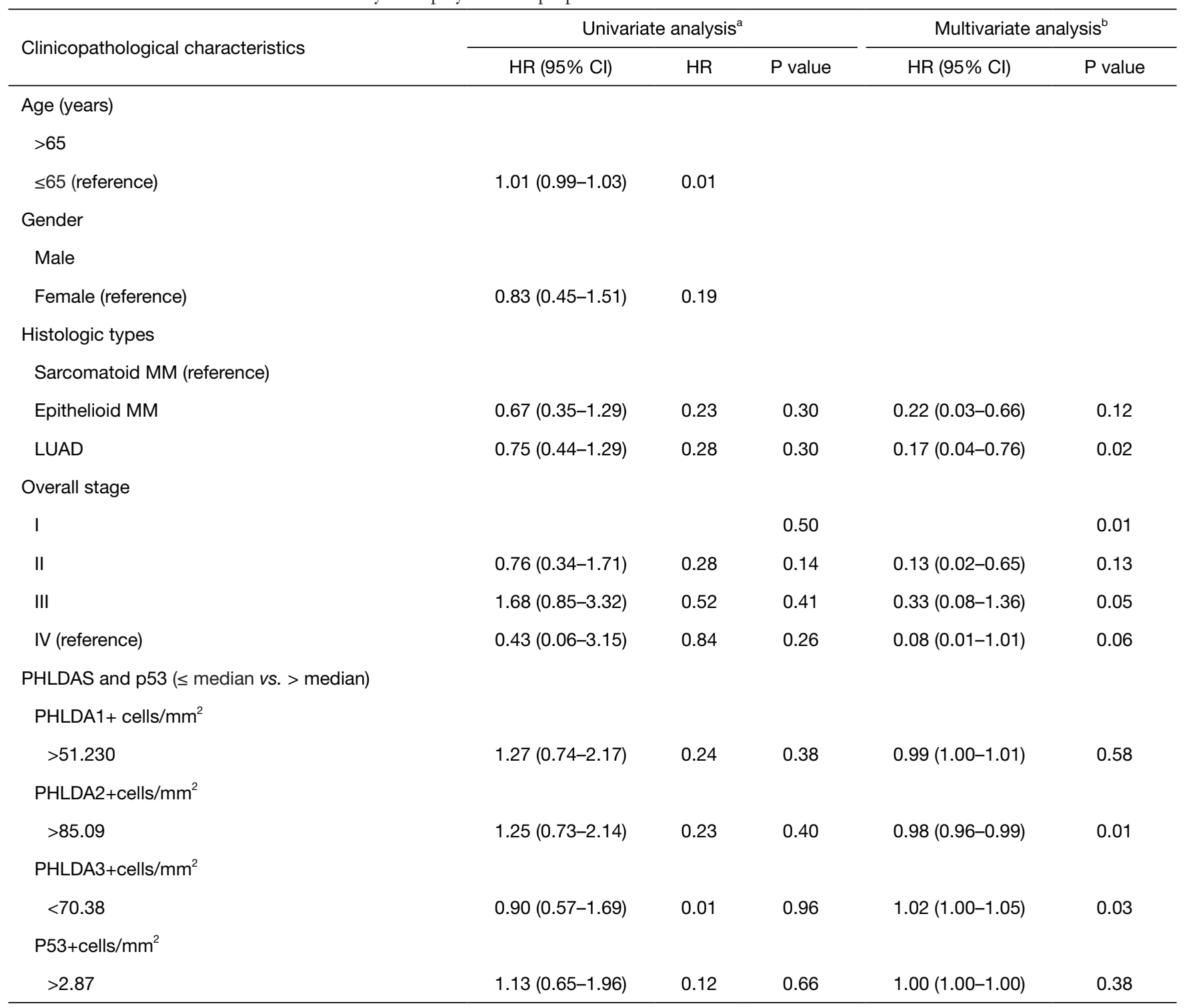

${ }^{a}$, univariate analysis was carried out without any adjustment in order to generate hazard ratios with confidence intervals for individual risk for each of the parameters on survival; ${ }^{b}$, multivariate analysis was carried out to analyze the effects of several risk parameters on survival. PHLDA, pleckstrin homology domain family $A$; HR, hazard ratio ( $\beta$ coefficient); $\mathrm{Cl}$, confidence interval; MM, malignant mesothelioma; LUAD, lung adenocarcinoma.

\section{Correlation between protein expression and clinical outcome}

The Cox model analysis of our cohort appears in Table 6 . While no statistical differences were found for any clinicopathologic parameters on the risk of death in univariate analysis, a multivariate Cox model analysis demonstrated that LUAD and epithelioid MM histotypes, and overall stage I were significantly related to low risk of death $(\mathrm{P}=0.02, \mathrm{P}=0.01, \mathrm{P}=0.01$, respectively). For the quantitative tumor staining of PHLDA2 and PHLDA3 staining, multivariate analysis showed a significant association with the risk of death $(\mathrm{P}=0.01$ and $\mathrm{P}=0.03)$. Patients that presented PHLDA2 $>85.09$ cells $/ \mathrm{mm}^{2}$ had a low risk of death $(\mathrm{HR}=0.98 ; 95 \% \mathrm{CI}$ : 0.96-0.99; $\mathrm{P}=0.01$ ) and median survival time of 48 months. In contrast, those with PHLDA3 $<70.38$ cells $/ \mathrm{mm}^{2}$ presented a high risk of death (HR $=1.02 ; 95 \%$ CI: $1.00-1.05 ; \mathrm{P}=0.03)$ and median survival time of 34 months. The multivariate Cox analysis 
confirmed that LUAD, epithelioid MM, overall stage I, reduced PHLDA2 protein expression, and increased PHLDA3 protein expression are independent risk factors.

\section{Discussion}

Based on our previous studies, we found that the PHLDA family acts as an immediate target downstream of the EGFR/ErbB2 signaling pathway in breast cancer $(6,26,27)$. Moreover, due to the fact that PHLDA genes are activated in various cancer types, the PHLDA family members have been increasingly perceived as promising targets for therapies against cancers (28). However, their detailed expression patterns, prognostic value, potential function, and drug interaction network in NSCLC, and especially in MM, remain largely unclear.

Several studies have reported decreased PHLDA1 mRNA expression in melanoma, breast carcinoma, oral carcinoma, gastric adenocarcinoma, and cholangiocarcinoma $(6,26)$. Interestingly, for PHLDA2 expression, studies on lung cancer have shown controversial reports: while Wang et al. (8) found increased levels of PHLDA2 in lung cancer, results from Hsu et al. (29) reached the opposite conclusion. In addition, in human neuroendocrine tumors, PHLDA3 was shown to act as a tumor suppressor gene in cases with increased PHLDA3 mRNA expression $(11,12)$. In agreement with these data, we noticed this variation of results during data mining, in which the data obtained in the TCGA for LUAD revealed significant mRNA expression of all members of the PHLDA family compared to normal tissue, and in Oncomine we observed underexpression of PHLDA1 mRNA and PHLDA2 mRNA overexpression, when compared to normal tissue. The same happened in the results for $M M$, where the GEO data showed lower expression of PHLDA1 and the expression of PHLDA3 significantly higher than normal samples, contrasting with the data from Oncomine in which both PHLDA1 and $P H L D A 3$ mRNA were not significantly expressed in a tumor tissue compared to normal samples.

Regarding the prognostic significance of PHLDA genes in NSCLC and MM, we observed that low PHLDA1 expression was responsible for shorter OS, FPS, and PPS in NSCLC; high PHLDA2 mRNA expression was significantly associated with better OS; and high PHLDA3 mRNA expression led to poorer OS. Using Mesothelioma (TCGA, Firehose Legacy) data, we demonstrated that patients with MM presenting high PHLDA1 and PHLDA2 mRNA expression had poor OS. In contrast, Muroi et al. (30) demonstrated that low expression of PHLDA3 is associated with poor outcome in patients with esophageal squamous cell carcinomas.

We also used the Comparative Toxicogenomics Database to show that a number of commonly used drugs were able to modulate PHLDAs. For example, while cisplatin may decrease PHLDA1 levels, a few carcinogenic substances, such as tetrachlorodibenzodioxin and benzo(a)pyrene, seem to increase its expression (Figure 4). Moreover, the protein-protein interaction network that resulted from the STRING analysis revealed intricate interrelationships among PHLDA family members (Figure 5). For instance, PHLDA1 acts on the Notch signaling pathway, PHLDA2 impacts cyclin-dependent protein serine/threonine kinase activity, and PHLDA3 relates to signal transduction by $\mathrm{p} 53$ class mediator. Thus, there is great relevance in the study of the PHLDA family members for future applications as we suggested in this study.

Additionally, to our knowledge, this is the first reported case of a PHLDAs protein investigation using a quantitative approach in NSCLC and MM. In our experimental study, we found that median protein expression for PHLDA1 was similar between LUAD and MM, whereas PHLDA2 and PHLDA3 protein levels were significantly higher in LUAD than in MM, though increased PHLDA3 protein levels were dependent on an advanced stage of the disease. P53 density was also higher in LUAD when compared with MM. Increasing evidence demonstrates that PHLDA protein levels, based on immunohistochemistry, were associated with diagnosis, prognosis, and targeted therapy in different cancer cells (31-35), including lung cancer (8). In addition, IHC staining markers were quantified using a cytoplasmic and nuclear algorithm to analyze PHLDAs. LUAD and MM showed predominant cytoplasmic staining of PHLDA1 and PHLDA2, while PHLDA3 staining demonstrated a strong and diffuse nuclear and cytoplasmic expression. Viúdez et al. (32) presented a strong correlation between disease free survival and PHLDA3 nuclear expression, where a higher expression of PHLDA3 was associated with worse clinical outcomes.

The multivariate Cox model analysis of our cohort showed that higher levels of PHLDA2 and PHLDA3 in patients with stage I and stage III LUAD were also significantly associated with risk of death. While patients with PHLDA2 $>85.09$ cells $/ \mathrm{mm}^{2}$ had lower risk of death and a median survival time of 48 months, those with PHLDA3 $<70.38$ cells $/ \mathrm{mm}^{2}$ had higher risk of death and a median survival time of 34 months. These inconsistent 
associations of PHLDAs expression levels with prognosis in lung cancer and MM between our study and previous studies might be due to differences in racial composition, population and sample size, and methods employed to measure the expression of PHLDAs.

Nevertheless, this study has some limitations. First, our research consists of an analysis based on previous data; therefore, additional experimental studies are still needed to confirm its results. Second, the clinical sample size of immunohistochemistry on tissue microarrays was not large enough and may have resulted in false negatives, a welldescribed problem in real-world oncology (36). Finally, as a retrospective cohort, none of our patients received a targeted therapy that evaluated the role of PHLDA in the therapeutic outcome. In summary, we suggest that more well-designed studies be carried out to support our findings.

However, the present study validated PHLDA1 underexpression in LUAD patients and its involvement on worse survival outcomes. On the other hand, both PHLDA2 and PHLDA3 were highly expressed in LUAD and MM according to our bioinformatics analysis and clinical tissue microarray. We therefore conclude that PHILDA family members might be adopted as promising predictive biomarkers and potential therapeutic targets in LUAD and MM.

\section{Acknowledgments}

We are grateful to Ms. Esmeralda Miristeni Eher and Ms. Sandra de Morais Fernezlian for their expertise on immunohistochemical protocols, and to Mr. Reginaldo Silva do Nascimento for his scanning expertise.

Funding: This work was supported by São Paulo Research Foundation (FAPESP) [2018/20403-6 to VLC]; the National Council for Scientific and Technological Development (CNPq) [483005/2012-6 to VLC]; and Coordenação de Aperfeiçoamento de Pessoal de Nível Superior - Brasil (CAPES) [Finance Code 001 to CMB].

\section{Footnote}

Reporting Checklist: The authors have completed the REMARK reporting checklist. Available at http://dx.doi. org/10.21037/jtd-20-2909

Peer Review File: Available at http://dx.doi.org/10.21037/jtd20-2909

Conflicts of Interest: All authors have completed the ICMJE uniform disclosure form (available at http://dx.doi. org/10.21037/jtd-20-2909). The authors have no conflicts of interest to declare.

Ethical Statement: The authors are accountable for all aspects of the work in ensuring that questions related to the accuracy or integrity of any part of the work are appropriately investigated and resolved. The study was conducted in accordance with the Declaration of Helsinki (as revised in 2013). The study was approved by the national ethics committee of $n^{\circ} 3.729 .622$ and individual consent for this retrospective analysis was waived.

Open Access Statement: This is an Open Access article distributed in accordance with the Creative Commons Attribution-NonCommercial-NoDerivs 4.0 International License (CC BY-NC-ND 4.0), which permits the noncommercial replication and distribution of the article with the strict proviso that no changes or edits are made and the original work is properly cited (including links to both the formal publication through the relevant DOI and the license). See: https://creativecommons.org/licenses/by-nc-nd/4.0/.

\section{References}

1. Goldstraw P, Chansky K, Crowley J, et al. The IASLC Lung Cancer Staging Project: Proposals for Revision of the TNM Stage Groupings in the Forthcoming (Eighth) Edition of the TNM Classification for Lung Cancer. J Thorac Oncol 2016;11:39-51.

2. Sugarbaker DJ, Flores RM, Jaklitsch MT, et al. Resection margins, extrapleural nodal status, and cell type determine postoperative long-term survival in trimodality therapy of malignant pleural mesothelioma: Results in 183 patients. J Thorac Cardiovasc Surg 1999;117:54-63.

3. Sugarbaker DJ, Strauss GM, Lynch TJ, et al. Node status has prognostic significance in the multimodality therapy of diffuse, malignant mesothelioma. J Clin Oncol 1993;11:1172-8.

4. Rusch VW, Venkatraman ES. Important prognostic factors in patients with malignant pleural mesothelioma, managed surgically. Ann Thorac Surg 1999;68:1799-804.

5. Frank D, Mendelsohn CL, Ciccone E, et al. A novel pleckstrin homology-related gene family defined by Ipl/ Tssc3, TDAG51, and Tih1: tissue-specific expression, chromosomal location, and parental imprinting. Mamm Genome 1999;10:1150-9.

6. Nagai MA. Pleckstrin homology-like domain, family 
A, member 1 (PHLDA1) and cancer. Biomed Rep 2016;4:275-81.

7. Kawase T, Ohki R, Shibata T, et al. PH domain-only protein PHLDA3 is a p53-regulated repressor of Akt. Cell 2009; 136:535-50.

8. Wang X, Li G, Koul S, et al. PHLDA2 is a key oncogeneinduced negative feedback inhibitor of EGFR/ErbB2 signaling via interference with AKT signaling. Oncotarget 2015;9:24914-26.

9. Fearon AE, Carter EP, Clayton NS, et al. PHLDA1 Mediates Drug Resistance in Receptor Tyrosine KinaseDriven Cancer. Cell Rep 2018;22:2469-81.

10. Li G, Wang X, Hibshoosh H, et al. Modulation of ErbB2 blockade in ErbB2-positive cancers: the role of ErbB2 Mutations and PHLDA1. PLoS One 2014;9:e106349.

11. Takikawa M, Ohki R. A vicious partnership between AKT and PHLDA3 to facilitate neuroendocrine tumors. Cancer Sci 2017;108:1101-8.

12. Ohki R, Saito K, Chen Y, et al. PHLDA3 is a novel tumor suppressor of pancreatic neuroendocrine tumors. Proc Natl Acad Sci U S A 2014;111:E2404-13.

13. Chandrashekar DS, Bashel B, Balasubramanya SAH, et al. UALCAN: A Portal for Facilitating Tumor Subgroup Gene Expression and Survival Analyses. Neoplasia 2017;19:649-58.

14. Danford T, Rolfe A, Gifford D. GSE: a comprehensive database system for the representation, retrieval, and analysis of microarray data. Pac Symp Biocomput 2008:539-50.

15. Suraokar MB, Nunez MI, Diao L, et al. Expression profiling stratifies mesothelioma tumors and signifies deregulation of spindle checkpoint pathway and microtubule network with therapeutic implications. Ann Oncol 2014;25:1184-92.

16. Rhodes DR, Yu J, Shanker K, et al. ONCOMINE: a cancer microarray database and integrated data-mining platform. Neoplasia 2004;6:1-6.

17. Rhodes DR, Kalyana-Sundaram S, Mahavisno V, et al. Oncomine 3.0: genes, pathways, and networks in a collection of 18,000 cancer gene expression profiles. Neoplasia 2007;9:166-80.

18. Győrffy B, Surowiak P, Budczies J, et al. Online Survival Analysis Software to Assess the Prognostic Value of Biomarkers Using Transcriptomic Data in Non-SmallCell Lung Cancer. PLoS One 2013;8:e82241

19. Goldman MJ, Craft B, Hastie M., et al. Visualizing and interpreting cancer genomics data via the Xena platform. Nat Biotechnol 2020;38:675-8.
20. Davis AP, Wiegers TC, Johnson RJ, et al. Text mining effectively scores and ranks the literature for improving chemical-gene-disease curation at the comparative toxicogenomics database. PLoS One 2013;8:e58201.

21. Shannon P, Markiel A, Ozier O, et al. Cytoscape: a software environment for integrated models of biomolecular interaction networks. Genome Res 2003;13:2498-504.

22. Szklarczyk D, Gable AL, Lyon D, et al. STRING v11: protein-protein association networks with increased coverage, supporting functional discovery in genomewide experimental datasets. Nucleic Acids Res 2019;47:D607-13.

23. Travis WD, Brambilla E, Nicholson AG, et al. The 2015 World Health Organization Classification of Lung Tumors: Impact of Genetic, Clinical and Radiologic Advances Since the 2004 Classification. J Thorac Oncol 2015;10:1243-60.

24. Travis WD, Brambilla E, Noguchi M, et al. International Association for the Study of Lung Cancer/American Thoracic Society/European Respiratory Society: International Multidisciplinary Classification of Lung Adenocarcinoma. J Thorac Oncol 2011;6:244-85.

25. Song X, Zhao C, Jiang L, et al. High PITX1 expression in lung adenocarcinoma patients is associated with DNA methylation and poor prognosis. Pathol Res Pract 2018;214:2046-53.

26. Bonatto N, Carlini MJ, de Bessa Garcia SA, Nagai MA. PHLDA1 (pleckstrin homology-like domain, family A, member 1) knockdown promotes migration and invasion of MCF10A breast epithelial cells. Cell Adh Migr 2018;12:37-46.

27. Nagai MA, Fregnani JH, Netto MM, et al. Downregulation of PHLDA1 gene expression is associated with breast cancer progression. Breast Cancer Res Treat 2007;106:49-56.

28. Fuselier TT, Lu H. PHLD Class Proteins: A Family of New Players in the p53 Network. Int J Mol Sci 2020;21:3543.

29. Hsu YL, Hung JY, Lee YL, et al. Identification of novel gene expression signature in lung adenocarcinoma by using next-generation sequencing data and bioinformatics analysis. Oncotarget 2017;8:104831-54.

30. Muroi H, Nakajima M, Satomura H, et al. Low PHLDA3 expression in oesophageal squamous cell carcinomas is associated with poor prognosis. Anticancer Res 2015;35:949-54.

31. Llamas-Velasco M, Pérez-Gónzalez YC, Bosch-Príncep 
$\mathrm{R}$, et al. Solid carcinoma is a variant of microcystic adnexal carcinoma: A 14-case series. J Cutan Pathol 2018:45:897-904.

32. Viúdez A, Carvalho FLF, Maleki Z, et al. A new immunohistochemistry prognostic score (IPS) for recurrence and survival in resected pancreatic neuroendocrine tumors (PanNET). Oncotarget 2016;7:24950-61.

33. Jeon SY, Kim DC, Song KH, et al. Expression Patterns of Gli-1, Pleckstrin Homology-Like Domain, Family A, Member 1, Transforming Growth Factor- $\beta 1 / \beta 2$, and p63 in Sebaceous and Follicular Tumors. Ann Dermatol 2014;26:713-21.

Cite this article as: Baldavira CM, Machado-Rugolo J, Prieto TG, Bastos DR, Balancin M, Ab'Saber AM, Yaegashi LB, Souza PC, Farhat C, Takagaki TY, Nagai MA, Capelozzi VL. The expression patterns and prognostic significance of pleckstrin homology-like domain family A (PHLDA) in lung cancer and malignant mesothelioma. J Thorac Dis 2021;13(2):689-707. doi: 10.21037/jtd-20-2909
34. Battistella M, Carlson JA, Osio A, et al. Skin tumors with matrical differentiation: lessons from hair keratins, beta-catenin and PHLDA-1 expression. J Cutan Pathol 2014;41:427-36.

35. Sellheyer K, Krahl D. PHLDA1 (TDAG51) is a follicular stem cell marker and differentiates between morphoeic basal cell carcinoma and desmoplastic trichoepithelioma. Br J Dermatol 2011;164:141-7.

36. Morris SM, Subramanian J, Gel ES, et al. Performance of next-generation sequencing on small tumor specimens and/or low tumor content samples using a commercially available platform. PLoS One 2018;13:e196556. 


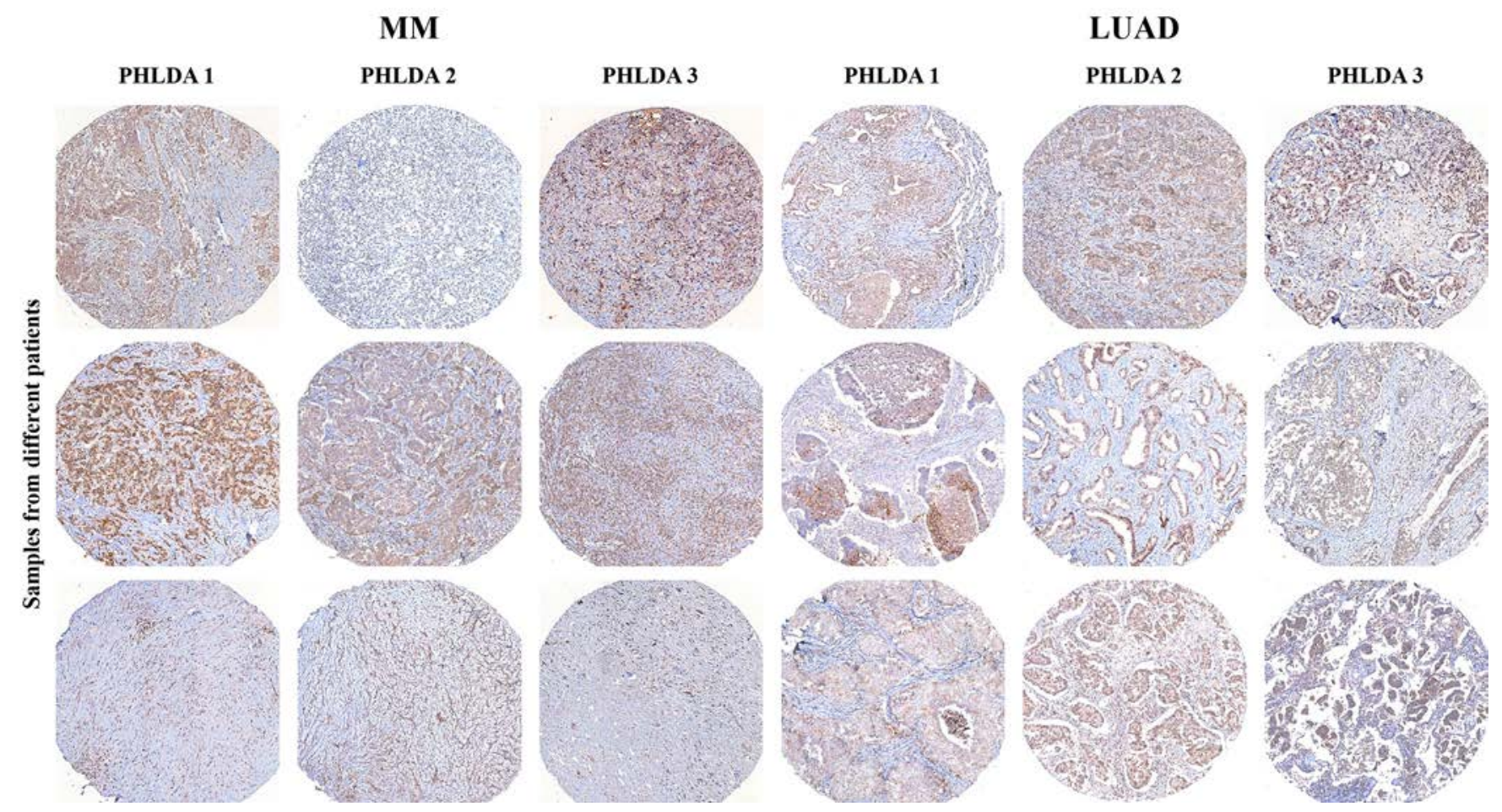

Figure S1 Expression of pleckstrin homology domain family A (PHLDA) family in malignant mesothelioma (MM) and lung adenocarcinoma (LUAD) tumors cores included in tissue microarray (TMA). Homogeneity observed between expression levels in different tumor cores. Original magnification $4 \times$. 


\section{Oncomine dataset}

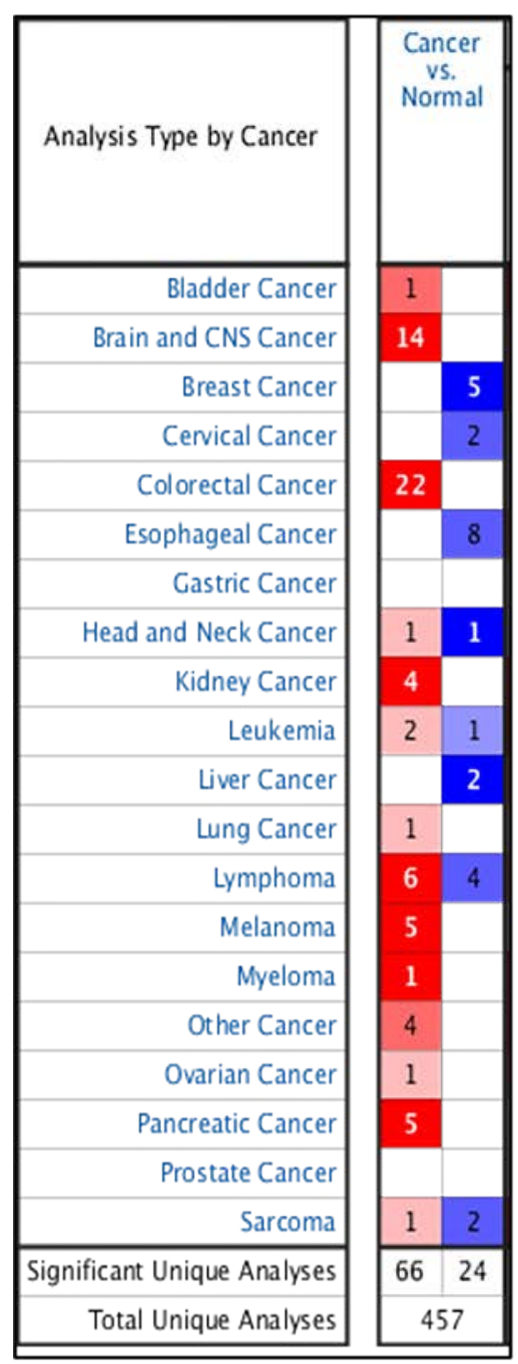

PHLDA1

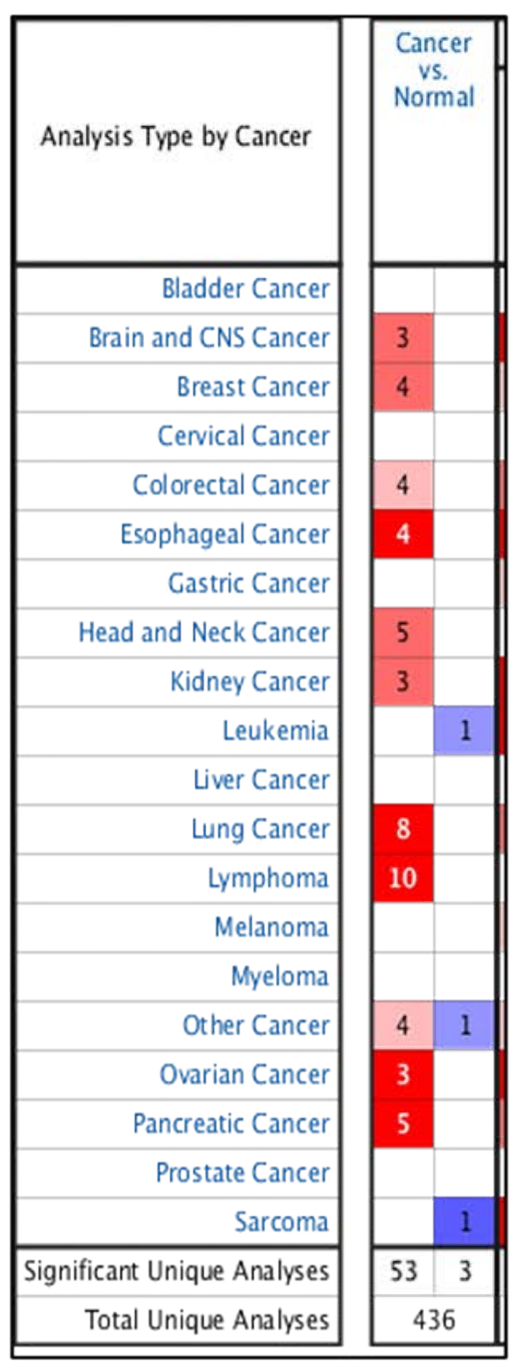

PHLDA2

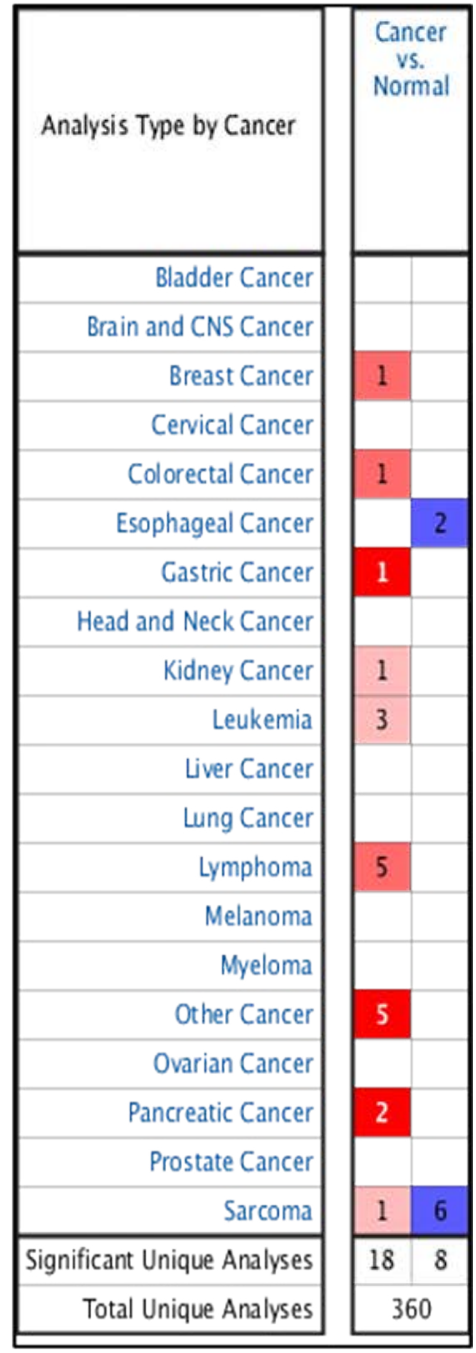

PHLDA3

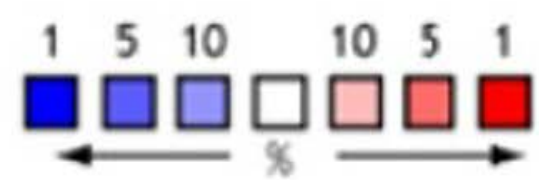

Figure S2 Oncomine analysis of PHLDA1, PHLDA2 and PHLDA3 mRNA expression in different types of cancer, showing the differences in expression levels between tumor and normal tissue. Red cells represent overexpression of genes in tumor tissue compared to normal, whereas blue cells indicate underexpression, satisfying the thresholds $\mathrm{P}<0.05$; fold change $\geq 1.5$; gene rank $\leq 10 \%$. PHLDA, pleckstrin homology domain family A. 


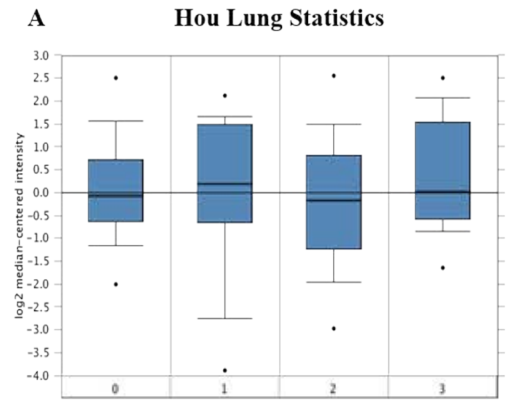

Legend

0 . Lung $(n=65)$

1. Large Cell Lung Carcinoma ( $\mathrm{n}=19) ; P=0.14 ; \mathrm{FC}=1.16$

2. Lung Adenocarcinoma $(\mathrm{n}=45) ; P=0.03 ; \mathrm{FC}=1.18$

3. Squamous Cell Lung Carcinoma $(\mathrm{n}=27) ; P=0.03 ; \mathrm{FC}=1.25$

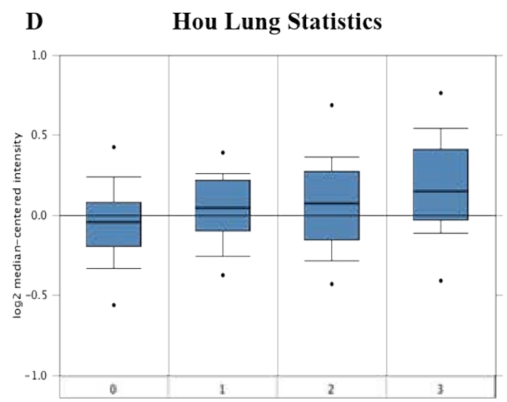

Legend

0 . Lung $(\mathrm{n}=65)$

1. Large Cell Lung Carcinoma $(\mathrm{n}=19) ; P=0.06 ; \mathrm{FC}=1.06$

2. Lung Adenocarcinoma $(\mathrm{n}=45) ; P<0.01 ; \mathrm{FC}=2.21$

3. Squamous Cell Lung Carcinoma ( $\mathrm{n}=27) ; P<0.01 ; \mathrm{FC}=1.173$

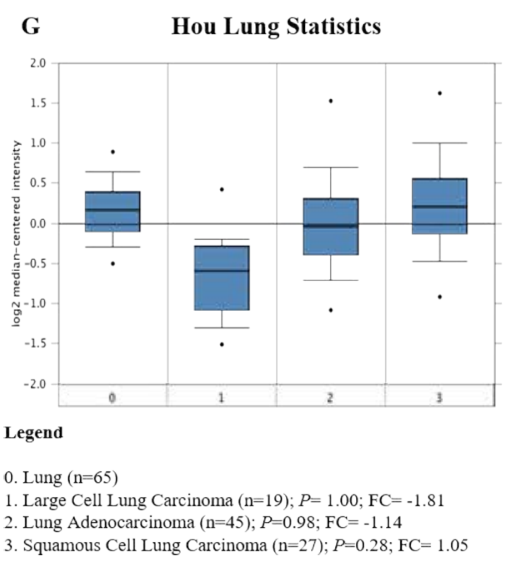

PHLDA1 Expression

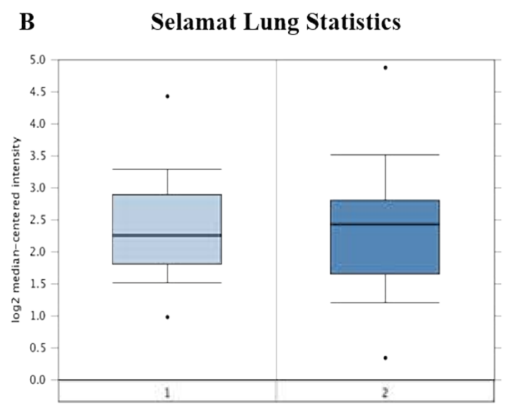

Legend

1. Lung $(\mathrm{n}=58)$

2. Lung Adenocarcinoma $(\mathrm{n}=58) ; P=0.61 ; \mathrm{FC}=-1.030$

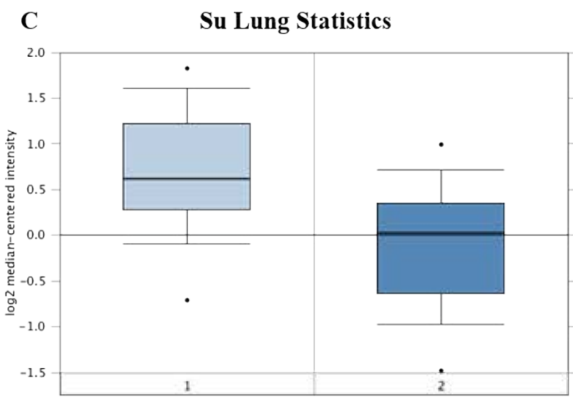

Legend

1. Lung $(\mathrm{n}=30)$

2. Lung Adenocarcinoma $(\mathrm{n}=27) ; P<0.01 ; \mathrm{FC}=-1.71$

\section{$\mathbf{E}$}

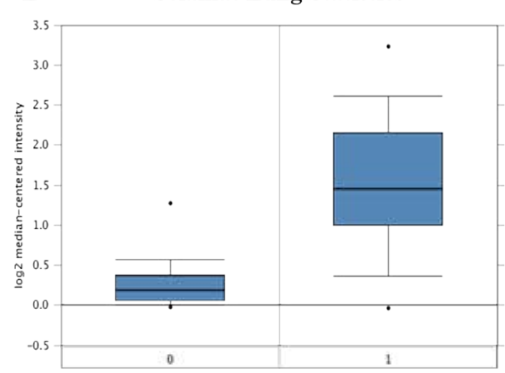

Legend

0. Lung $(\mathrm{n}=58)$

1. Lung Adenocarcinoma $(\mathrm{n}=58) ; P<0.01 ; \mathrm{FC}=2.39$

PHLDA3 Expression
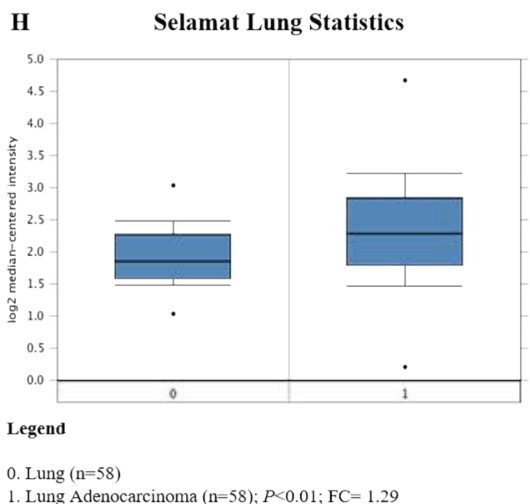

F

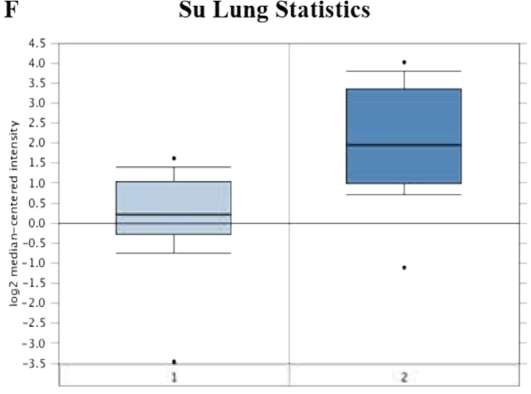

Legend

1. Lung $(\mathrm{n}=30)$

2. Lung Adenocarcinoma ( $\mathrm{n}=27) ; P<0.01 ; \mathrm{FC}=3.63$

Figure S3 Oncomine analysis of PHLDA1, PHLDA2 and PHLDA3 expression in NSCLC and their corresponding normal tissues. (A) In the Hou Lung and (B) Selamat datasets, there was no apparent change in PHLDA1 gene expression in lung adenocarcinoma (LUAD) (fold change $=1.18$ and -1.03 , respectively), whereas in the $(\mathrm{C})$ Su dataset, there was a trend for underexpression of PHLDA1 in LUAD tissue (fold change $=-1.71$ ). PHLDA2 was overexpressed according to (D) Hou Lung (fold change = 2.21), (E) Selamat (fold change =2.39), and (F) Su (fold change $=3.63$ ) datasets. No changes were found in the expression levels of PHLDA3 in LUAD according to (A) Hou Lung (fold change $=-1.14$ ), (B) Selamat (fold change $=1.29$ ) and (C) Su (fold change =1.03) datasets. PHLDA, pleckstrin homology domain family A. 
PHLDA Expression in Gordon Mesothelioma

Grouped by Cancer Type

PHLDA1 Expression

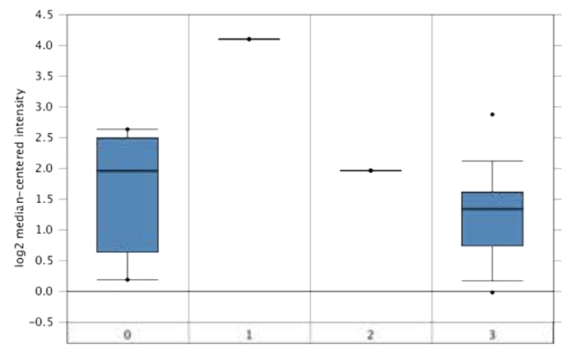

A Legend

0 . No value $(\mathrm{n}=54)$

1. Lung $(\mathrm{n}=4)$

3. Pleural Malignant Mesothelioma ( $\mathrm{n}=40) ; P=0.70 ; \mathrm{FC}=-1.09$
PHLDA2 Expression

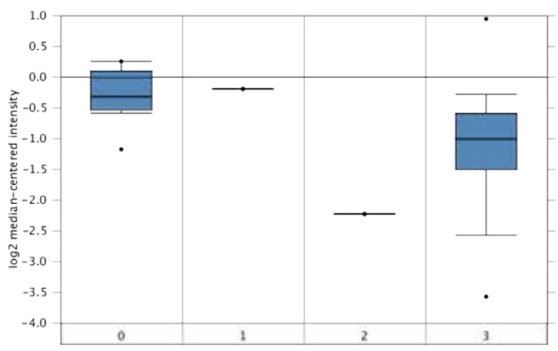

B Legend

0 . No value $(\mathrm{n}=10)$

1. Mesothelioma $(\mathrm{n}=1)$

2. Pleural Biphasic Mesothelioma $(\mathrm{n}=1)$

3. Pleural Malignant Mesothelioma ( $\mathrm{n}=42$ ): $P<0.01 ; \mathrm{FC}=-1.92$
PHLDA3 Expression

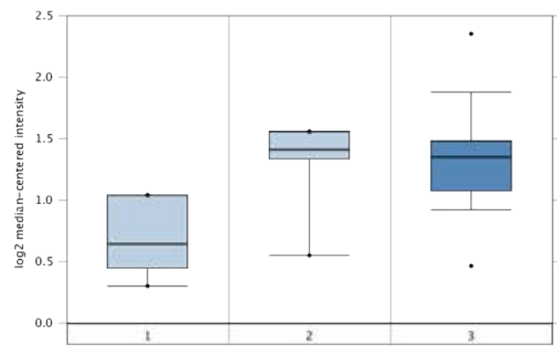

C Legend

1. Lung $(\mathrm{n}=4)$

2. Pleura $(\mathrm{n}=5)$

3. Pleural Malignant Mesothelioma ( $\mathrm{n}=40) ; P=0.04 ; \mathrm{FC}=1.25$

Figure S4 PHLDA gene expression in malignant mesothelioma (MM) according to the Gordon Mesothelioma dataset. PHLDA1 (A) and PHLDA3 (C) were not significantly expressed in tumors compared to normal tissue (fold change $=-1.09$ and 1.25 , respectively), (B) PHLDA2 was underexpressed in MM (fold change=-1.92). The $\mathrm{X}$-axis of the plot represents normal vs. pleural malignant mesothelioma, the $\mathrm{Y}$-axis represents mRNA expression in $\log 2$ median-centered intensity; the line in the middle represents the median value. $\mathrm{P}<0.05$ was considered significant. PHLDA, pleckstrin homology domain family A. 


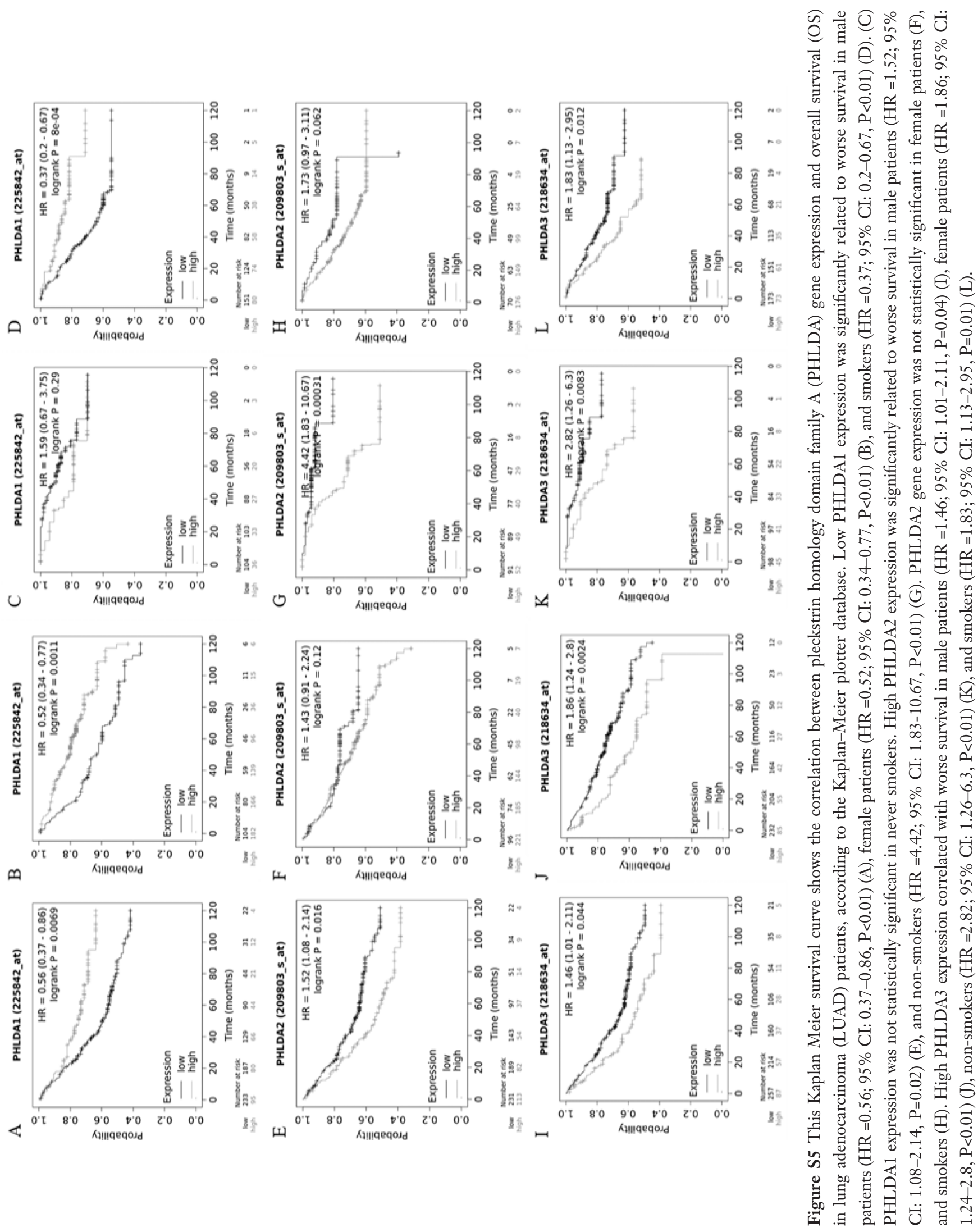


Table S1 Variables associated with overall survival (OS) of 87 patients diagnosed with malignant mesothelioma

\begin{tabular}{|c|c|c|c|c|}
\hline Variables & \multicolumn{2}{|c|}{ Univariate } & \multicolumn{2}{|c|}{ Multivariate } \\
\hline Age & $0.76(0.3-1.8)$ & 0.53 & & \\
\hline Sex (Male vs. Female) & $0.90(0.5-1.6)$ & 0.73 & & \\
\hline TP53 (Mutated vs. Wild type) & $1.04(0.5-2.0)$ & 0.92 & & \\
\hline Stage & & 0.77 & & \\
\hline Stage (II vs. I) & $0.64(0.3-1.5)$ & 0.31 & & \\
\hline Stage (III vs. I) & $0.78(0.4-1.6)$ & 0.51 & & \\
\hline Stage (IV vs. I) & $0.71(0.3-1.7)$ & 0.44 & & \\
\hline PHLDA2 (high vs. low) & $1.87(1.2-3.0)$ & $<0.01$ & $1.71(1.1-2.7)$ & 0.03 \\
\hline
\end{tabular}

Univariate and multivariate analysis employed a Cox proportional hazards model using the Mesothelioma TCGA Firehose Legacy database by UCSC Xena tool. HR, hazard ratio; $95 \% \mathrm{Cl}$, 95\% confidence intervals. 